\title{
ACTITUDES HACIA LA DEMOCRACIA EN ESPAÑA: LEGITIMIDAD, DESCONTENTO Y DESAFECCIÓN ${ }^{1}$
}

\author{
José Ramón Montero \\ Universidad Autónoma de Madrid
}

Richard Gunther

Ohio State University

Mariano Torcal

Universidad Autónoma de Madrid

\section{RESUMEN}

Este artículo examina las principales actitudes hacia la democracia en España durante las dos últimas décadas. Se han seleccionado numerosos indicadores empíricos para diferenciar tres facetas que suelen considerarse indistintamente, y por lo tanto confundirse de forma sistemática: la legitimidad democrática, el descontento político y la desafección política. En el artículo se analiza su evolución respectiva desde la transición democrática, y se mantiene que pertenecen a dimensiones conceptuales y empíricas diferentes. Para ello se incluyen, además, los resultados de dos tipos de pruebas: un análisis factorial confirma el distinto agrupamiento de los indicadores en el nivel individual, y un análisis de cohortes identifica pautas diferenciadas de continuidad y cambio en las distintas generaciones.

Este artículo trata de las percepciones básicas sobre la democracia en España durante los últimos veinte años. En él queremos continuar examinando las pautas culturales por las que el ciudadano se relaciona con el sistema político; por lo

${ }^{1}$ Versiones anteriores de este artículo se presentaron en la Conferencia Internacional sobre The erosion of confidence in advanced democracies (Society of Comparative Research y Université Libre de Bruxelles, Bruselas, noviembre de 1996) y en el Seminario sobre Cultura política en las sociedades democráticas (Fundación Pablo Iglesias, Madrid, enero de 1997). Queremos agradecer la ayuda prestada por Justin Byrne, Anna Mélich y Modesto Escobar, la traducción de Jesús Cuéllar del texto original en inglés, las facilidades del Centro de Estudios Avanzados en Ciencias Sociales, del Instituto Juan March, y la financiación de la Comisión Interministerial de Ciencia y Tecnología (CICYT [SEC95-1007]). 
tanto, este artículo se asienta en los trabajos anteriores que analizaban la generación de actitudes de apoyo a la democracia española (Montero y Gunther, 1994), apuntaban peculiaridades esenciales en el marco general de su cultura política (Montero y Torcal, 1990), comparaban los niveles de la legitimidad democrática en las nuevas democracias del sur de Europa (Montero y Morlino, 1993; Morlino y Montero, 1995) y destacaban las diferentes dimensiones existentes en las culturas políticas de esas nuevas democracias (Torcal, 1995). Con el español de nuevo como caso empírico, queremos ahora plantearnos algunas distinciones básicas que la literatura sobre el tema no suele tomar en consideración, lo que, a nuestro juicio, ocasiona problemas importantes en el desarrollo de los conceptos relacionados con dimensiones actitudinales y supone una seria deficiencia de la teoría empírica de la democracia. Más particularmente, discutimos aquí algunas extendidas suposiciones sobre el apoyo democrático y la satisfacción con el sistema político en cuanto componentes claves de lo que Kaase y Newton (1995) han agrupado bajo el epígrafe de "teorías de la contradicción, la crisis y la catástrofe». Siguiendo estos enfoques teóricos y empíricos, cuestionamos la relación que suele establecerse entre las actitudes fundamentales hacia la democracia (ya sean la legitimidad, el apoyo, la confianza o cualquier otro término parecido) y las evaluaciones específicas sobre la actuación del sistema democrático. Una definición tout court de la legitimidad como satisfacción con la democracia supone, entre otras cosas, que las variaciones en el grado de ésta pueden interpretarse como amenazas a la estabilidad de aquélla, una conclusión que subyace en muchas de las teorías que abordan de un modo u otro los problemas actuales de la democracia. En el otro extremo, señalamos también que la utilización de un concepto demasiado amplio de satisfacción con la democracia tiende a confundir las percepciones manifestadas en diferentes niveles del sistema político, las evaluaciones de muchos objetos políticos y, sobre todo, las orientaciones afectivas de los ciudadanos para con su sistema político.

En este artículo mantenemos que tanto las distinciones entre legitimidad democrática y satisfacción con la democracia, de un lado, y entre esta última y la desafección política, de otro, son significativas en términos conceptuales y resultan empíricamente comprobables. En nuestra opinión, esas distinciones son relevantes para la teoría de la democracia de países con sistemas políticos muy arraigados, así como para las teorías que pretenden explicar procesos recientes de transiciones políticas desde regímenes autoritarios. De modo similar, defendemos también que, en el marco de las orientaciones y actitudes de los ciudadanos hacia los sistemas democráticos, estas tres familias de conceptos se corresponden con datos empíricos diferentes. Además, a lo largo del tiempo las tres pueden desarrollarse de forma asimismo diferente, y seguir pautas de transmisión intergeneracional muy diversas.

Hemos seleccionado el caso español para nuestro análisis, y a él se refiere la mayor parte de los datos empíricos que utilizamos. Hay tres razones que hacen del español un caso especialmente interesante. En primer lugar, por motivos temporales. Es evidente que España pertenece a ese amplio grupo de países que disfrutan de democracias consolidadas, y que también forma parte de la tercera ola 
de democratización de los años setenta (Huntington, 1991; Linz y Stepan, 1996). En consecuencia, muchos de los enfoques teóricos que se han desarrollado recientemente para explicar los problemas de la democracia pueden aplicarse sólo parcialmente al caso español. Por ejemplo, el régimen autoritario franquista estaba aún en el poder cuando aparecieron las primeras obras sobre la crisis financiera del Estado (O'Connor, 1973) y los problemas de la legitimidad democrática (Habermas, 1975). El inicio de la transición coincidió con los primeros análisis de los fenómenos de sobrecarga del Estado (King, 1975) y de crisis de la democracia (Crozier et al., 1975). Y el proceso que condujo a la aprobación de una Constitución democrática y a la consolidación del nuevo sistema político tuvo lugar cuando los estudiosos comenzaban a discutir los problemas de gobernabilidad y las amenazas implícitas en los nuevos movimientos sociales (Rose, 1980; Dalton et al., 1984). Esta divergencia temporal permite reexaminar los anteriores estudios a la luz de las percepciones y actitudes de los españoles, y comprobar las peculiaridades de su formación y evolución a lo largo del tiempo.

La segunda razón tiene que ver con la variabilidad: durante los últimos veinte años, los españoles han hecho frente a una amplia gama de situaciones políticas diferentes. Entre ellas se encuentran los episodios finales de uno de los más duraderos regímenes autoritarios europeos, los riesgos de la transición política, los problemas de consolidación de una democracia joven, las dificultades de reconstrucción de un Estado sobre la base de una estructura territorial completamente nueva, un sistema de partidos con fases agudas de inestabilidad, la incertidumbre tras la alternancia en el poder (que, inmediatamente después de un intento de golpe de Estado, llevó al poder a un partido socialdemócrata por primera vez en la historia de España), crisis económicas recurrentes en el marco de las cifras de desempleo más altas de Europa, y una sucesión de crisis políticas provocadas por escándalos en la financiación de los partidos, por casos de corrupción de cargos públicos y por revelaciones sobre comportamientos ilegales en la lucha contra el terrorismo. Si se tiene en cuenta la intensidad de estas experiencias y el período relativamente corto en el que han ocurrido, es razonable suponer que hayan tenido una influencia no pequeña en el modo como los ciudadanos perciben el sistema político, evalúan su rendimiento y desarrollan vínculos afectivos con sus diversos componentes. Finalmente, la tercera razón se refiere a la disponibilidad de una notable cantidad de datos de encuestas, lo cual hace del caso español un laboratorio especialmente útil para comprobar la evolución de las orientaciones y actitudes hacia el sistema político.

El artículo está dividido en cinco partes. Las tres primeras se ocupan, respectivamente, de los indicadores de legitimidad democrática, descontento político y desafección política. En ellas analizamos el significado de los diferentes indicadores y su evolución durante las dos últimas décadas, y esperamos demostrar que pertenecen a dimensiones diferentes. En la cuarta sección examinamos los resultados de un análisis factorial que nos permite comprobar hasta qué punto esos tres indicadores están interrelacionados en el nivel indivi- 
dual. Y en la última sección presentamos los datos de un análisis de cohortes mediante el cual se identifican los elementos de cambio y continuidad que confirman la diferente naturaleza de cada uno de los tres tipos de indicadores.

\section{LOS NIVELES DE LEGITIMIDAD DEMOCRÁTICA}

Frente a quienes defienden que pueden hacer falta décadas para el desarrollo de actitudes de apoyo a un sistema político (véase, por ejemplo, Pridham, 1995), una considerable mayoría de españoles ha apoyado decididamente el régimen democrático, especialmente desde 1982. Esto se demuestra tanto en su comportamiento electoral como en la evolución de sus actitudes hacia la democracia. En el ámbito electoral, y dejando al margen el caso del País Vasco, los partidos antisistema o/y antidemocráticos han disfrutado de escasos apoyos. En las elecciones generales de 1977 sólo obtuvieron el 0,61 por 100 de los votos. En 1979, cuando comenzaban a aparecer síntomas de un cierto desencanto entre los votantes y las élites políticas, los partidos de extrema derecha recibieron unos 400.000 votos (el 2,3 por 100 de los emitidos); en 1982, los partidos extremistas habían desaparecido prácticamente de la escena política. En las elecciones generales de 1996, los partidos antidemocráticos (fuera del País Vasco) recibieron sólo 17.500 votos, es decir, un 0,5 por 100 del total.

Los indicadores actitudinales de la legitimidad democrática que se derivan de los datos de encuestas concuerdan con esta pauta del comportamiento electoral. Concebimos la legitimidad como una actitud positiva de los ciudadanos hacia las instituciones democráticas, consideradas como la forma de gobierno más apropiada ${ }^{2}$. Este concepto es relativo, ya que ningún sistema es completamente legítimo para todos los ciudadanos, y la intensidad del apoyo positivo a sus instituciones cambia según las personas. Por consiguiente, la legitimidad puede ser considerada como «la creencia de que las instituciones políticas existentes, a pesar de sus defectos y fallos, son mejores que otras que pudieran haber sido establecidas" (Linz, 1988, 65; 1978a, 16). Esta definición es también relativa en la medida en que se relaciona con el convencimiento de que el sistema democrático es la menos mala de las formas de gobierno. Como también ha escrito Linz $(1978 b, 18)$, «en última instancia, la legitimidad democrática se basa en la creencia de que para un determinado país y en un momento dado, ningún otro tipo de régimen podría asegurar un mayor éxito de los objetivos colectivos» ${ }^{3}$. Las tablas 1 y 2 muestran dos indicadores que reflejan las

${ }^{2}$ Utilizamos aquí un concepto minimalista de legitimidad, ya que creemos que es la mejor manera de resolver los habituales problemas de medición y operacionalización del concepto; unos problemas que, además, se han agravado a causa de su carácter multidimensional; véanse Morlino y Montero (1995, 232), Linz (1988, 62) y McDonough, Barnes y López Pina (1986, 737).

3 Véanse también Linz y Stepan (1996, 76 y ss.), Diamond y Lipset (1995), Diamond (1998) y, desde una perspectiva muy diferente, Rawls (1993, 137). Para un amplio análisis desde la teoría política, véase Beetham (1991). 
percepciones fundamentales sobre la legitimidad de la democracia española. La tabla 1 presenta el porcentaje de encuestados que se mostraba de acuerdo con la afirmación de que «la democracia es el mejor sistema para un país como el nuestro", y la tabla 2 contiene la distribución de opiniones de quienes están a favor de un sistema democrático en cualquier circunstancia y de aquéllos que apoyarían, en ciertas situaciones, un régimen autoritario. Las conclusiones son inequívocas. En ambos casos, los españoles respaldan mayoritariamente la democracia: entre dos terceras y más de tres cuartas partes de los encuestados estaban de acuerdo con la afirmación que considera que la democracia es superior a cualquier otro sistema político (tabla 1). El grado de apoyo extraordinariamente alto que recibe la democracia en 1978 indica probablemente un efecto luna de miel (por el que los españoles dieron un mayoritario voto de confianza a las instituciones democráticas desde el momento en que se constituyeron [Weil, 1989]). Aunque la firmeza de estas actitudes sufrió después un cierto debilitamiento, todos los datos existentes confirman que se ha mantenido un alto grado de legitimidad democrática desde 1982. La tabla 2 subraya esta tendencia al aportar también datos del escaso apoyo recibido por las alternativas antidemocráticas durante todo este período ${ }^{4}$. Incluso entre los votantes del principal partido conservador, Alianza Popular (desde 1989, Partido Popular $[\mathrm{PP}])$, que fue fundado por destacadas personalidades del régimen de Franco, el número de partidarios de la democracia en cualquier circunstancia sobrepasa con creces al de quienes estarían a favor de una alternativa autoritaria en ciertas situaciones (Montero, 1993; Montero y Gunther, 1994). Estos datos contradicen la tesis que propugna que la cultura política española no es intrínsecamente democrática, o que contiene reductos significativos de sentimientos antidemocráticos (como señala, por ejemplo, Wiarda, 1989, x y 2).

De hecho, el grado de apoyo a la democracia en España resulta similar al de los restantes países europeos occidentales 5 . Una encuesta de 1992, que utiliza el mismo indicador (véase la tabla 3), pone de manifiesto que los niveles de apoyo a la democracia en tres de los cuatro países del sur de Europa eran similares o superiores a los de la Unión Europea. Sólo Italia se aparta de esta pauta, pero su 72 por 100 no es muy diferente del promedio comunitario (78 por 100). No obstante, los países del sur de Europa mostraban algunas peculiaridades. Es significativo el contraste que hay entre el acusado aumento del apoyo a la democracia en Portugal entre 1985 y 1992 y el mantenimiento del alto índi-

${ }^{4}$ Aunque Fuchs, Guidorossi y Svensson $(1995,348)$ y Tóka $(1995,359)$, entre otros, han señalado que el indicador utilizado en la tabla 2 se refiere a una idea abstracta de democracia, es evidente que los entrevistados no conciben la democracia de forma separada de su propia situación, país o experiencia histórica, y aún menos la consideran como un concepto abstruso, teórico e inaplicable. En realidad, creemos que éste es un indicador válido de las actitudes de los ciudadanos hacia el sistema político, ya que recalca la importancia del nivel sistémico y, a la vez, resulta fácilmente comprensible por su imbricación con la experiencia diaria e histórica del entrevistado y porque le permite elegir entre diferentes respuestas. Véase Muller, Jukam y Seligson (1982).

${ }^{5}$ Las raíces de la legitimidad en la transición española son analizadas en Maravall (1995, 257 y ss.), Aguilar (1996, 209 y ss.) y Montero y Torcal (1990). 


\section{TABLA 1}

La democracia como mejor sistema para España, 1978-1994

\begin{tabular}{|c|c|c|}
\hline La democracia es el mejor sistema para un país como el nuestro & $\%$ & $(N)$ \\
\hline $1978 \ldots \ldots \ldots . . .$. & 77 & 5.898 \\
\hline (n) & 69 & 3.132 \\
\hline . & 81 & 1.703 \\
\hline 等, & 74 & 5.463 \\
\hline 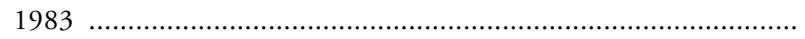 & 73 & 5.481 \\
\hline 1988 & 87 & 4.548 \\
\hline 1993 & 79 & 1.448 \\
\hline 1994 & 82 & 2.491 \\
\hline
\end{tabular}

Fuentes: Centro de documentación de DATA, para 1978-1993, y Banco de Datos del Centro de Investigaciones Sociológicas (CIS), para 1994.

ce de apoyo registrado en Grecia. Los dos países con menores índices relativos de apoyo a la democracia, España e Italia, son también aquéllos en los que la preferencia por regímenes autoritarios es mayor, aunque en ambos sólo una parte muy pequeña de la población expresa estos sentimientos (Morlino y Montero, 1995; Montero y Gunther, 1994). Además, la validez de este indicador se refuerza con los datos procedentes de algunos países del cono sur latinoamericano ${ }^{6}$. Como también puede verse en la tabla 3, lejos de registrarse la unanimidad casi total que cabría esperar si los encuestados se refirieran a una concepción abstracta e idealizada de la democracia, los ciudadanos expresan preferencias diferentes en cada país. Los casos de Uruguay y Argentina, donde los niveles de apoyo a sus respectivos regímenes democráticos son similares a los de los países de Europa occidental, contrastan vivamente con los de Chile y Brasil, que se enfrentan a situaciones muy difíciles. Finalmente, al igual que ha ocurrido en el sur de Europa, en estos países latinoamericanos, con la excepción de Chile y, en menor medida, Brasil, se ha registrado un incremento del apoyo a la democracia.

${ }^{6}$ E incluso por los datos existentes en otros contextos, como, por ejemplo, Corea del Sur. La aplicación de un indicador idéntico en 1996 dio como resultado un 65 por 100 de apoyo a la democracia y un 17 por 100 a un régimen autoritario; en 1997 los porcentajes fueron, respectivamente, 67 y 23 por 100. Pueden verse Shin y Shyu (1997), Shin y Rose (1997) y, más en general, Rose y Shin (1997). Para un examen sistemático de este indicador y otros similares, véase Diamond (1998). 
TABLA 2

La legitimidad de la democracia en España, 1980-1996

(en porcentajes)

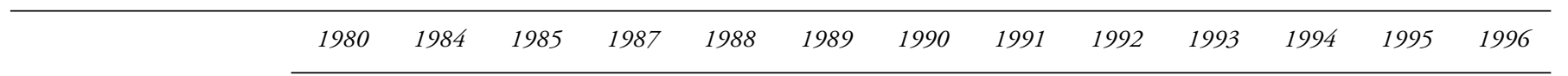

La democracia es preferible a cualquier otra forma de gobierno 49

69

70

71

72

68

80

76

73

81

73

79

81

un régimen autoritario,

una dictadura, puede ser

preferible al sistema

democrático .....................

A la gente como yo, lo

mismo le da un sistema

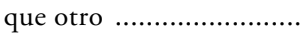

No sabe, no contesta

8

33

$11 \quad 10$

12

10

10

7

10

12

7

$\begin{array}{ccc}8 & 9 & 8 \\ 10 & 8 & 7 \\ 9 & 4 & 4 \\ (2.491) & (2.478) & (2.481)\end{array}$

Fuente: Banco de Datos, CIS. 


\section{TABLA 3}

Legitimidad democrática en Europa occidental (1985-1995) y en algunos paises latinoamericanos (1988-1996) (en porcentajes)

\begin{tabular}{|c|c|c|c|c|c|}
\hline \multicolumn{2}{|c|}{ Paises } & Democracia & Autoritarismo & Indiferencia & $N S / N C$ \\
\hline \multicolumn{6}{|c|}{ Europa Occidental, 1992} \\
\hline \multirow{2}{*}{\multicolumn{2}{|c|}{$\begin{array}{l}\text { Dinamarca } \\
\text { Luxemburgo }\end{array}$}} & 92 & 4 & 2 & 1 \\
\hline & & 82 & 2 & 6 & 9 \\
\hline \multicolumn{2}{|c|}{ Alemania } & 81 & 8 & 7 & 3 \\
\hline \multicolumn{2}{|c|}{ 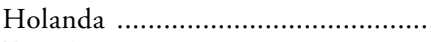 } & 81 & 9 & 5 & 5 \\
\hline \multicolumn{2}{|c|}{ 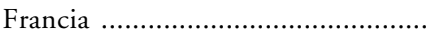 } & 78 & 7 & 11 & 5 \\
\hline \multicolumn{2}{|c|}{ 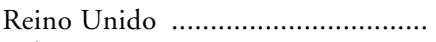 } & 76 & 6 & 11 & 6 \\
\hline \multicolumn{2}{|c|}{ Bélgica .......................................... } & 70 & 10 & 10 & 10 \\
\hline \multicolumn{2}{|c|}{ 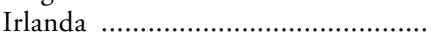 } & 63 & 10 & 21 & 6 \\
\hline \multicolumn{6}{|c|}{ Sur de Europa } \\
\hline \multirow[t]{3}{*}{ Grecia } & 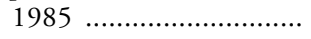 & 87 & 5 & 6 & 2 \\
\hline & $1988 \ldots \ldots \ldots \ldots \ldots \ldots \ldots \ldots \ldots . .$. & 90 & 3 & 4 & 3 \\
\hline & 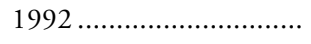 & 91 & 4 & 3 & 2 \\
\hline \multirow[t]{3}{*}{ Portugal } & 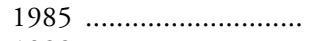 & 61 & 9 & 7 & 23 \\
\hline & 1988 & 84 & 7 & 9 & - \\
\hline & 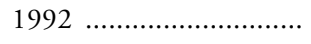 & 83 & 9 & 4 & 4 \\
\hline \multirow[t]{4}{*}{ España } & $1985 \ldots \ldots \ldots \ldots \ldots \ldots \ldots \ldots$ & 70 & 10 & 9 & 11 \\
\hline & $1988 \ldots \ldots \ldots \ldots \ldots \ldots \ldots \ldots \ldots$ & 75 & 8 & 14 & 3 \\
\hline & 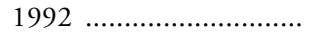 & 78 & 9 & 7 & 6 \\
\hline & 1995 …....................... & 79 & 9 & 8 & 4 \\
\hline \multirow[t]{4}{*}{ Italia } & 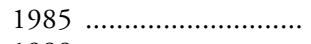 & 70 & 13 & 10 & 7 \\
\hline & 1988 & 74 & 13 & 13 & - \\
\hline & $1992 \ldots \ldots \ldots \ldots \ldots \ldots \ldots \ldots . .$. & 73 & 14 & 8 & 7 \\
\hline & 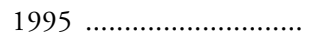 & 79 & 9 & 8 & 4 \\
\hline \multicolumn{6}{|c|}{ América Latina } \\
\hline \multirow[t]{2}{*}{ Uruguay } & $1988 \ldots \ldots \ldots \ldots \ldots \ldots \ldots \ldots \ldots$ & 73 & 10 & 8 & 9 \\
\hline & 1995 .............................. & 80 & 8 & 6 & 6 \\
\hline \multirow[t]{3}{*}{ Argentina } & 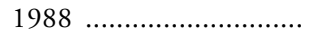 & 74 & 13 & 10 & 3 \\
\hline & 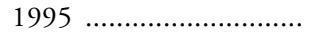 & 77 & 11 & 6 & 6 \\
\hline & 1996 ............................... & 71 & 15 & 11 & 3 \\
\hline \multirow[t]{3}{*}{ Chile } & 1988 & 57 & 11 & 27 & 5 \\
\hline & 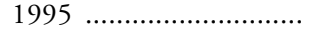 & 52 & 18 & 25 & 4 \\
\hline & 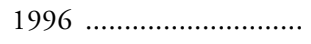 & 54 & 19 & 23 & 4 \\
\hline \multirow[t]{3}{*}{ Brasil } & 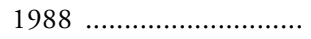 & 43 & 21 & 26 & 10 \\
\hline & 1995 ............................ & 41 & 21 & 23 & 15 \\
\hline & 1996 & 50 & 24 & 21 & 5 \\
\hline
\end{tabular}

Fuentes: Para 1985 en el sur de Europa y España en 1995, Banco de Datos del CIS; para 1988 y 1992, Eurobarómetro, 30, 1988, y 37, 1992. Para Italia en 1994, los datos han sido cedidos amablemente por Paolo Segatti y el Archivio Ricerche Demoscopiche, Universidad de Pavía. Para los países latinoamericanos en 1988, Moisés (1995, 160); para 1995, Linz y Stepan $(1996,222)$, y para 1996, Lagos (1997, 133). 


\section{EL DESCONTENTO POLÍTICO: EFICACIA DEL SISTEMA Y SATISFACCIÓN}

En contraste con la continuidad existente en estos indicadores de la legitimidad de la democracia española, las evaluaciones del rendimiento de su sistema político y de sus élites gobernantes han oscilado de forma considerable a lo largo del tiempo. La idea más extendida sobre las relaciones entre ambos indicadores mantiene que las fluctuaciones en el grado de satisfacción del ciudadano con la democracia y/o con el gobierno son significativas y pueden amenazar la estabilidad del mismo sistema democrático, dado que están directamente relacionadas con el funcionamiento del nuevo régimen. Aquí defendemos, por el contrario, que los regímenes democráticos pueden mantener su estabilidad incluso cuando se enfrentan a niveles elevados de insatisfacción con el sistema. Dicho de otro modo, su pervivencia descansa más en las actitudes hacia la legitimidad que en la satisfacción o en la percepción que se tenga de su eficacia.

Antes de analizar este punto con mayor detenimiento, quizás sea necesario ocuparse de dos cuestiones referidas a la posibilidad de separar los conceptos de legitimidad y de eficacia. Algunos autores han dudado de la capacidad de los ciudadanos para distinguir entre ambas dimensiones (Muller y Jukam, 1977; Kinder y Sears, 1985, 725), y sostienen que en los análisis muestrales las respuestas obtenidas a las preguntas sobre la «legitimidad» de un sistema pueden estar muy influidas por la valoración que les merecen a los encuestados las autoridades políticas, por su evaluación de la actuación de las instituciones políticas o por el desfase existente entre la realidad e importantes valores abstractos $^{7}$. Otros autores, cuyos análisis se basan principalmente en datos muestrales que sólo contienen medidas de insatisfacción con la democracia o con el funcionamiento de sus instituciones, defienden que aquéllas son indicadores apropiados y suficientes para medir el apoyo al sistema "con un grado de generalización relativamente bajo» (Fuchs, Guidorossi y Svensson, 1995, 330); o señalan que esos factores equivalen a medidas de la legitimidad (Fuchs y Klingemann, 1995, 425; Tóka, 1995, 359); o aceptan que el apoyo político se defina simplemente como satisfacción con la democracia (Anderson y Guillory, 1997, 70); o deciden equiparar la legitimidad con una concepción amplia de confianza que incluye un continuo desde la esfera privada a la pública (McDonough, Barnes y López Pina, 1994, 370). Por nuestra parte, mantenemos, en primer lugar, que la legitimidad y la eficacia son distintas no sólo desde un punto de vista conceptual, sino también empírico. Esta distinción ha sido ana-

Para lo que es ya una discusión clásica de la confianza (trust), un concepto equivalente en términos funcionales, véanse Miller (1974a y 1974b) y Citrin (1974); para una revisión teórica y un análisis empírico de datos alemanes, véase Gabriel (1996); y para un análisis empírico, obtenido en los resultados de un estudio piloto que defiende la distinción entre lo que se denomina confianza basada en los cargos (incumbent-based trust) y confianza basada en el régimen (regimebased trust), véase Craig, Niemi y Silver (1990). 
lizada desde diversas perspectivas y con diferentes consecuencias teóricas ${ }^{8}$ y, si se dispone de los indicadores apropiados, puede demostrarse empíricamente. En términos generales, la eficacia del sistema y la satisfacción política pueden concebirse como componentes de un síndrome más amplio de descontento político, definido como la expresión de una cierta frustración que surge de comparar lo que uno tiene y lo que debería tener (Gamson, 1968; López Pintor, 1997) ${ }^{9}$. La eficacia del sistema comprende una serie de percepciones relacionadas con la eficiencia del mismo a la hora de resolver problemas básicos (Dahl, 1971, 144); en otras palabras, con su capacidad para solucionar problemas que los ciudadanos consideran de especial importancia (Morlino y Montero, 1995, 234). Y la insatisfacción política (que se utiliza con más frecuencia que su antónimo) expresa el desagrado que produce un objeto social o político significativo, y puede estimarse, en consecuencia, como un rechazo general de algo que no responde suficientemente a los deseos de los ciudadanos (Di Palma, 1970, 30). Por lo tanto, la insatisfacción política surge de la evaluación que hacen los ciudadanos del rendimiento del régimen o de las autoridades, así como de los resultados políticos que generan (Farah, Barnes y Heunks, 1979). En este artículo, las dos dimensiones seleccionadas como indicadores de la insatisfacción política son el binomio gobierno/oposición (es decir, en qué medida el apoyo o la oposición a las autoridades políticas existentes influyen en la valoración que los ciudadanos hacen del rendimiento del gobierno) y la apreciación de las políticas públicas (esto es, hasta qué punto los ciudadanos evalúan el desfase que existe entre sus propias preferencias sobre esas políticas y los resultados surgidos de las que realmente se implementan) ${ }^{10}$.

En segundo lugar, defendemos también que a los ciudadanos de los países que han experimentado recientemente transiciones desde regímenes autoritarios (como los del sur de Europa) les resulta sumamente fácil distinguir entre legitimidad y eficacia. La experiencia personal directa del autoritarismo y, en todo caso, la socialización política hacen posible que los entrevistados distingan entre gobierno autoritario y democrático, y que puedan separar sus evaluaciones sobre el rendimiento del sistema (satisfacción) de su apoyo al actual

8 Además de las obras clásicas de Easton (1965 y 1975), véanse, por ejemplo, los puntos de vista contrapuestos de Dahrendorf (1980) y Offe (1984). Lipset (1981), Linz (1978a y 1978b), Morlino (1985) y Di Palma (1990), entre otros, han realizado interesantes análisis de los conceptos de eficacia, eficiencia y rendimiento del régimen. La distinción entre actitudes que confieren legitimidad a un régimen y valoración de la eficacia del rendimiento del gobierno ha sido también analizada en diversos estudios recientes de varios países europeos. Véanse, por ejemplo, Morlino y Montero (1995), Weil (1989), Kuechler (1991), Finkel, Muller y Seligson (1989) y Fuchs (1992).

9 Para un sugestivo análisis de un concepto parecido, el de decepción, véase Hirschman (1982).

${ }^{10}$ Farah, Barnes y Heunks (1979, 429 y ss.) añaden a estos dos indicadores los relativos a la eficacia política interna y externa, que para nosotros constituyen - y así esperamos demostrarlo más adelante- dimensiones del concepto de desafección política. Véase también Miller (1979, 964 y ss.). 
régimen democrático (legitimidad). Como consecuencia de sus memorias individuales o colectivas, los europeos del sur están mejor pertrechados cultural y actitudinalmente para distinguir entre la legitimidad de un régimen y las percepciones de su eficacia. Sin duda, la capacidad de los griegos, portugueses y españoles para hacer estas distinciones es diferente en cada generación y disminuirá a medida que el paso del tiempo vaya haciendo menos relevante la experiencia del autoritarismo para la memoria colectiva del país. Pero desde finales de los setenta hasta mediados de los noventa dicha memoria aún era intensa y significativa para muchos ciudadanos, bien que fuera menor en Italia que en los otros tres países. Por el contrario, en los países con democracias muy arraigadas es mucho más difícil para los encuestados valorar sus sistemas políticos comparándolos con una hipotética (y apenas imaginable) opción no democrática: en estas circunstancias las preguntas acerca de regímenes políticos alternativos podrían resultar abstractas e irreales (Morlino y Montero, 1995; McDonough, Barnes y López Pina, 1986 y 1994, y Weil, 1989). En estas democracias, las medidas de legitimidad podrían confundirse más fácilmente con las evaluaciones de la eficacia del sistema o del rendimiento político ${ }^{11}$.

Algunas características del caso español facilitan el análisis de estas dos dimensiones y determinan el impacto en ellos de variaciones en las condiciones económicas, sociales y políticas. Para empezar, la situación económica ha sufrido cambios considerables durante el período aquí estudiado. En contraste con los altos índices de crecimiento económico y de aumento del nivel de vida individual producidos en los últimos quince años del régimen autoritario franquista, los procesos de transición y consolidación democrática tuvieron lugar en medio de crisis económicas sucesivas, agravadas por las distintas «crisis del petróleo» de los años setenta. Como en el resto de los países industrializados, la economía española tocó fondo en 1981-1982, cuando el desempleo llegó al 20 por 100 de la población activa (García Delgado, 1990). Por el contrario, durante la segunda mitad de los ochenta los indicadores económicos mejoraron sustancialmente y, aunque el índice de paro siguió siendo el más alto de Europa occidental, los niveles generales de riqueza aumentaron de forma llamativa. La súbita y grave recesión que comenzó a principios de los años noventa, cuando el paro sobrepasó el 23 por 100, constituyó un segundo desafío económico. En 1993 el clima de crisis económica llegó a su punto álgido, pero a partir del siguiente comenzó a observarse una fuerte recuperación.

Las percepciones sobre el rendimiento de los gobiernos españoles en asuntos no económicos también fluctuaron considerablemente durante el período. Gran parte del éxito de la transición a la democracia se achacó a los gobiernos

${ }_{11}$ Naturalmente, esta capacidad para distinguir entre diferentes regímenes y, por tanto, para juzgarlos existe también en Europa oriental; véanse Linz y Stepan (1996, 437 y ss.), Rose y Haerpfer (1992, 44 y ss.), Mishler y Rose (1996), Rose y Mishler (1996) y Rose (1997). Para el distinto caso de Corea del Sur o Taiwan, pueden verse Shin y Shyu (1997) y, más generalmente, Diamond (1998). 
de Unión de Centro Democrático (UCD), lo que permitió a su presidente, Adolfo Suárez, capitalizar en las elecciones anticipadas de marzo de 1979 la ola de satisfacción que siguió a la aprobación de la nueva Constitución, en diciembre de 1978. Poco después, sin embargo, el apoyo popular a la gestión de UCD se vino abajo. Se estimaba que sus débiles y divididos Gobiernos eran incapaces de hacer frente a los retos planteados por la crisis económica, el aumento de la violencia terrorista y una política autonómica errática (Gunther, 1986). También se temía que la ineficacia de los gobiernos centristas estuviera socavando gravemente la legitimidad inicialmente otorgada al sistema democrático. Este diagnóstico se resumió en el término desencanto, que reflejaba la desilusión a la que se había llegado tras las grandes expectativas surgidas al comienzo de la transición desde el autoritarismo; un fenómeno que por lo demás parece darse en todas las transiciones democráticas (O’Donnell y Schmitter, 1986, 56; Huntington, 1991, 230). En general, se afirmaba que el desencanto amenazaba la consolidación del nuevo régimen. Sin embargo, estos temores se desvanecieron después de las elecciones generales de 1982, que posibilitaron por vez primera una mayoría parlamentaria absoluta, llevaron al poder al Partido Socialista Obrero Español (PSOE) y facilitaron la recuperación económica. A finales de los años ochenta, el índice de crecimiento económico de España era el segundo de Europa, la inflación había descendido de forma significativa y un Gobierno socialista muy estable había alcanzado notables éxitos tanto en política exterior como interior. El segundo período de descontento comenzó a principios de los noventa y se reflejó en percepciones muy negativas de la crisis económica y en opiniones cada vez más críticas sobre los escándalos políticos relacionados con la financiación de los partidos, la corrupción de algunos altos cargos de la administración socialista y el descubrimiento de los delitos cometidos en la lucha contra el terrorismo de ETA (Wert, 1996). La recuperación económica de mediados de los noventa y la victoria electoral del Partido Popular en 1996 estuvieron acompañadas por una notable mejora en las evaluaciones del rendimiento político.

Los datos empíricos disponibles reflejan esta evolución. Como puede verse en el gráfico 1a, existe una covariación casi perfecta entre el grado de satisfacción con la situación económica y la valoración de las condiciones políticas, y ambos factores corren parejos a las cambiantes circunstancias antes apuntadas ${ }^{12}$. Según cabía esperar, la insatisfacción con la situación económica ha sido más acusada precisamente en los peores momentos de las dos recesiones. Pero resulta un tanto sorprendente comprobar que las valoraciones de la situación política siguen exactamente la misma pauta. Además, otras dos evaluaciones diferentes de eficacia del sistema (la creencia de que la «democracia

${ }^{12}$ Las preguntas tenían la siguiente formulación: «En términos generales, ¿diría usted que la situación política [económica] en España es muy buena, bastante buena, ni buena ni mala, bastante mala o muy mala?» En el gráfico 1a, las valoraciones positivas incluyen «muy buena» y «bastante buena». 
permite la solución de los problemas de los españoles» y, de modo general, la «satisfacción con el funcionamiento de la democracia en España») evolucionaron de forma paralela a las valoraciones de la situación económica y política (gráfico $1 \mathrm{~b})^{13}$.

\section{GRÁFICO 1a}

Situaciones politicas y económicas en España: la evolución de las valoraciones positivas, 1976-1996

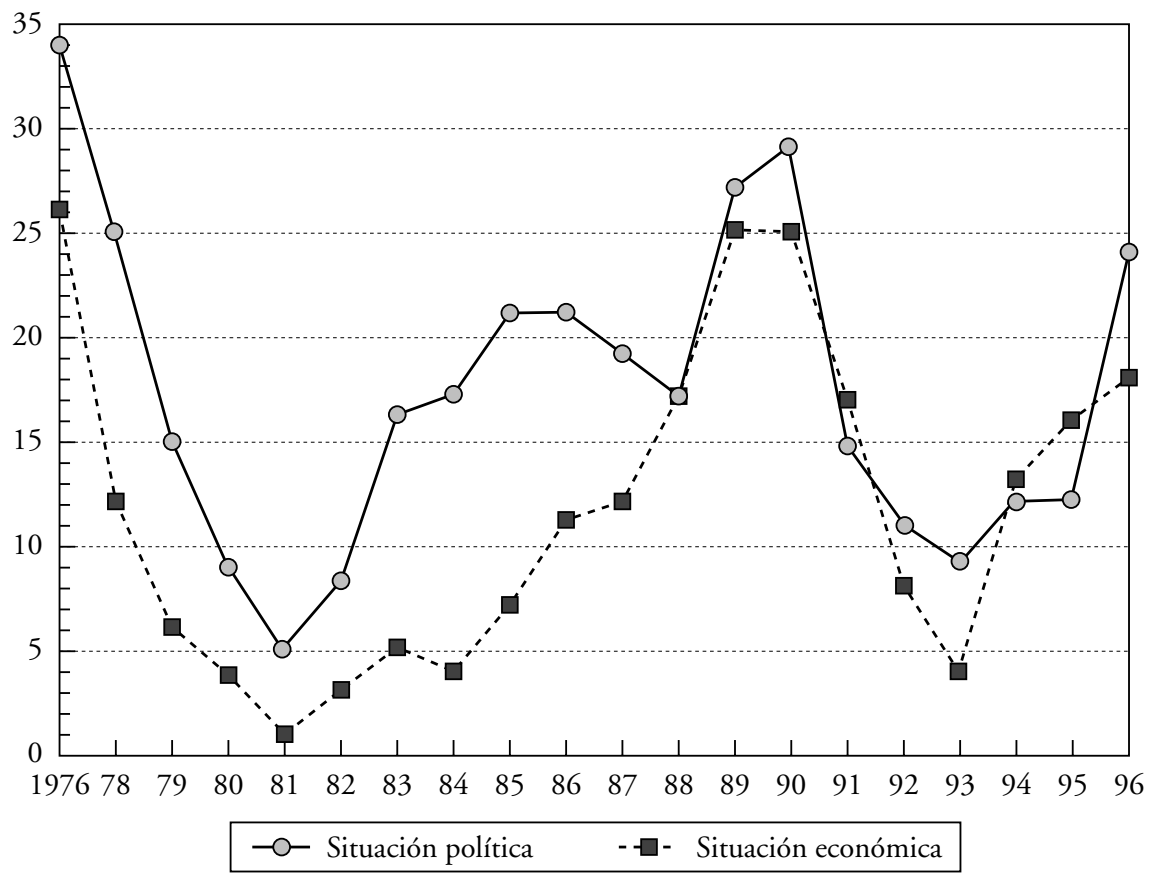

Fuentes: Banco de Datos del CIS y Archivo de datos de Demoscopia.

${ }_{13}$ El gráfico $1 \mathrm{~b}$ utiliza las preguntas habituales del Centro de Investigaciones Sociológicas (CIS) y del Eurobarómetro referidas a la satisfacción: «En conjunto, ¿está usted muy satisfecho, bastante satisfecho, no muy satisfecho o nada satisfecho con el funcionamiento de la democracia en España?» Hay que señalar que el grado general de satisfacción con la democracia en España es comparable a la media europea, y considerablemente más alto que en Italia; sus tendencias han sido también similares. Pueden verse Kuechler (1991), Fuchs, Guidorossi y Svensson (1995), Morlino y Tarchi (1996) y Anderson y Guillory (1997). 


\section{GRÁFICO 1b}

Eficacia del sistema (1978-1994) y satisfacción con la democracia (1983-1995)
en España

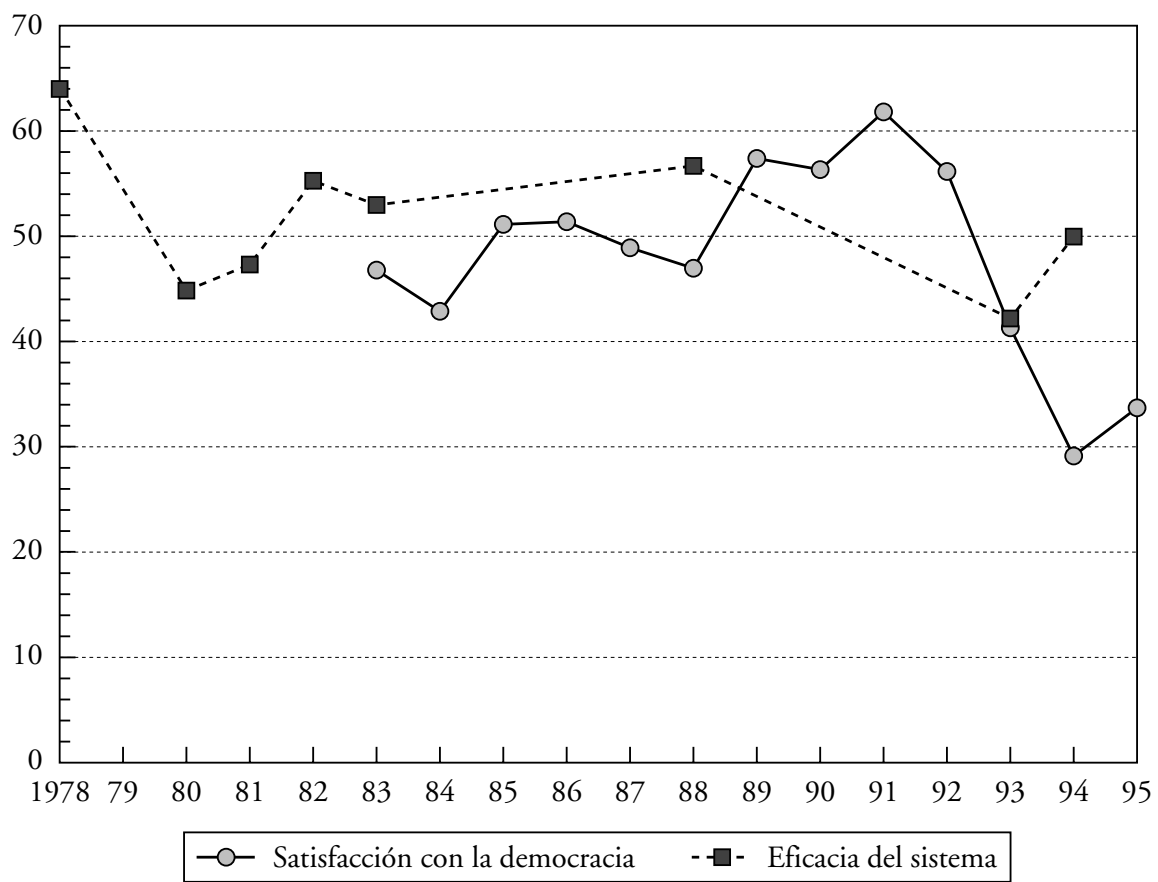

FUENTES: Para la eficacia del sistema, Centro de documentación de DATA, para 1978-1993, y Banco de Datos del CIS, para 1994. Para la satisfacción con la democracia, Eurobarómetro, Trends 1974-94, y 43, 1995, y Banco de Datos del CIS, para 1978-1984.

Estos datos temporales ponen de manifiesto la covariación existente entre las cuatro variables de satisfacción/eficacia del sistema. Independientemente de cómo esté redactada la pregunta, estos indicadores muestrales parecen estar refiriéndose a la misma dimensión. Pero mientras que los encuestados tienen aparentemente dificultades para distinguir entre su evaluación del gobierno, las condiciones económicas y la eficacia del sistema político en la resolución de problemas básicos, parecen diferenciar nítidamente estas valoraciones de sus opiniones sobre la legitimidad del régimen democrático. Como hemos visto anteriormente, los niveles de apoyos actitudinales tanto a la democracia como a su alternativa autoritaria se han mantenido prácticamente constantes, y no se han visto afectados por las crisis económicas de principios de los ochenta y de los noventa, por el generalizado descontento con los Gobiernos de UCD antes 
de su desastre electoral de 1982, o por los escándalos que rodearon al Gobierno socialista en los años que precedieron a su derrota electoral de 1996. Estas pautas subrayan tres puntos básicos. El primero es que las actitudes relacionadas con la satisfacción/eficacia del sistema tienen un componente significativo de "partidismo" y están muy centradas en las actividades del gobierno ${ }^{14}$. El segundo es que la insatisfacción con las políticas públicas, que, como ya sabemos, es la otra dimensión básica de la insatisfacción política (Farah, Barnes y Heunks, 1979), se halla en gran medida determinada por el estado de la economía; pero no siempre ocurre así1 ${ }^{15}$. El caso español demuestra que la economía política de las actitudes relativas a la satisfacción con el rendimiento democrático sólo tiene consecuencias limitadas (Clarke, Dutt y Kornberg, 1993; Linz y Stepan, 1996, 76-81). Finalmente, la legitimidad fundamental de la democracia, tanto desde un punto de vista teórico como empírico, es relativamente autónoma del descontento político, es decir, de las percepciones sobre la ineficacia del sistema y la insatisfacción con la democracia. En consecuencia, un nivel relativamente alto de legitimidad puede aislar al régimen del impacto negativo que de otra forma las crisis económicas o políticas podrían tener en su estabilidad (Finkel, Muller y Seligson, 1989; Morlino y Montero, 1995).

Estas conclusiones tienen cierta importancia para los análisis que sugieren que las dificultades políticas y económicas (sobre todo, en las nuevas democracias) pueden tener una influencia negativa inmediata en el apoyo al régimen. Frente a las concepciones deterministas de la relación entre apoyo a la democracia, eficacia del sistema y satisfacción con el mismo, creemos que esa relación es bastante más compleja. De acuerdo con Linz y Stepan (1996, 229), Maravall $(1995,276)$ y Diamond (1998, 42 ss.), rechazamos que los indicadores de legitimidad vayan siempre estrechamente unidos y estén relacionados causalmente con la satisfacción respecto al estado de la economía. Esas conclusiones tienen también implicaciones relevantes para los estudios que señalan (por ejemplo, Fuchs y Klingemann, 1995, 440) que la legitimidad de las democracias occidentales depende cada vez más de sus rendimientos económicos. En el caso de España, las críticas a la ineficacia del sistema o la insatisfacción con el funcionamiento de la democracia reflejan claramente un desacuerdo partidista y/o ideológico con las medidas del gobierno (Montero y Gunther, 1994); pero la legitimidad democrática no se ha visto inevitablemente socavada por el descontento económico, el pesi-

14 Para datos comparativos acerca de la relación entre insatisfacción y voto contra el partido del gobierno, véanse Schmitt (1983), Fuchs, Guidorossi y Svensson (1995, 344-347) y Anderson y Guillory (1977), que también analizan el impacto que tienen algunas instituciones políticas básicas sobre la satisfacción.

15 Véanse Kuechler (1991), Finkel et al. (1989), Weil (1989) y Linz y Stepan (1996, 81). Estos estudios subrayan que el apoyo a la democracia en España aumentó a pesar de los graves problemas derivados del rendimiento político de los gobiernos a principios de los años ochenta. 
mismo político, los escándalos políticos y otros aspectos impopulares de las actividades gubernamentales. Es posible que estos factores hayan alimentado procesos de desalineamientos partidistas y derrotas de los partidos en el gobierno, pero ni ellos mismos per se ni, en el peor de los casos, la degeneración de la política partidista han aumentado de forma significativa el respaldo a las alternativas antidemocráticas (véase también Maravall y Santamaría, 1989).

Un factor adicional que debilita los vínculos entre rendimiento económico y apoyo a la democracia consiste en que este último puede verse afectado por factores de naturaleza política, y más particularmente por distintas dimensiones de la actuación del gobierno, como son el respeto a las libertades fundamentales y al ordenamiento jurídico (Diamond y Lipset, 1995; Fuchs, 1992). También el paso del tiempo favorece la institucionalización de la legitimidad democrática, ya que ayuda a aislar los apoyos al régimen de los problemas coyunturales en la situación económica e incluso de las crisis económicas, así como de los escándalos políticos. Además, la opinión pública puede darse cuenta de que lo que los gobiernos son capaces de realizar resulta muy limitado, y puede también tomarse sus promesas con escepticismo; en definitiva, puede estar preparada para sus fracasos (Kaase y Newton, 1995, 75). El conocimiento pragmático por parte de los ciudadanos de que algunos problemas sociales quizá sean insolubles o rebasen la capacidad de cualquier líder político para afrontarlos con algún tipo de éxito puede, asimismo, limitar los términos por los que la insatisfacción con el rendimiento del sistema político termine socavando los apoyos actitudinales básicos de la democracia; como se ha demostrado para algunos países de la Europa del Este, ese pragmatismo llega a cristalizar incluso en una suerte de paciencia política, por medio de la cual los ciudadanos prevén que la solución de determinados problemas puede necesitar varios años (Rose y Mishler, 1997). Por último, el hecho de que en los regímenes democráticos el gobierno sea siempre pro tempore puede desempeñar un papel decisivo a la hora de hallar salida a situaciones problemáticas: unas nuevas elecciones, y la posibilidad de que llegue al poder otro partido, producen generalmente consecuencias positivas para las valoraciones de la democracia.

\section{LA DESAFECCIÓN POLÍTICA}

Hasta ahora hemos señalado que la insatisfacción política o económica y las percepciones de la ineficacia del sistema forman parte del campo del descontento político, que a su vez es diferente del de la legitimidad democrática. A continuación nos ocuparemos de otra dimensión actitudinal, relacionada con orientaciones o actitudes políticas negativas que parecen estar muy enraizadas en las culturas políticas y que son extraordinariamente importantes. Según la 
distinción hecha por Di Palma (1970, 30), estas actitudes pueden integrar un fenómeno de desafección, es decir, un cierto alejamiento o desapego de los ciudadanos con respecto a su sistema político. La desafección política es un concepto tan crecientemente utilizado como diversamente definido. Si se considera como una especie de síndrome, sería posible situar a sus sintomas en un continuo que fuera desde un polo positivo de ciudadanos completamente integrados y con fuertes sentimientos de cercanía a su sistema político, que pasara a través de puntos intermedios caracterizados por un cierto desapego respecto a elementos significativos del régimen, y que alcanzara un polo negativo definido por una hostilidad completa hacia el sistema político y un consecuente alejamiento del mismo. Entre los síntomas más importantes de esta gradación se encontrarían el desinterés, la ineficacia, la disconformidad, el cinismo, la desconfianza, el distanciamiento, la separación, el alejamiento, la impotencia, la frustración, el rechazo, la hostilidad y la alienación. Se trata, por lo tanto, de una familia de conceptos diversos que capta unas orientaciones básicas hacia el sistema político cuyo denominador común radica en "la tendencia a la aversión de su componente afectivo» (Citrin, 1972, 92; véanse también Citrin y Elkins, 1975; Di Palma, 1970, y Abramson, 1983). El término asociado por lo general con desafección es alienación, bien que sean conceptos diferentes: mientras que el último se refiere a un sentimiento persistente de extrañamiento respecto a las instituciones, valores y líderes políticos existentes, y que tiene como consecuencia que los ciudadanos se consideren a sí mismos forasteros o intrusos, el primero alude a un conjunto de sentimientos mucho más difuso, y por el que los asuntos políticos son vistos como algo lejano, faltos de importancia o carentes de sentido (Citrin et al., 1975, 2-3).

De otra parte, también deberíamos distinguir en el ámbito político entre desafección y descontento o insatisfacción. Nuestra hipótesis es que la desafección política consiste en un conjunto de actitudes básicas hacia el sistema político que son diferentes de las que componen la insatisfacción política y la legitimidad democrática. En modo similar a como Pye $(1971,157)$ vinculaba alienación y socialización, la insatisfacción política puede considerarse como el resultado de la divergencia entre los valores generalmente positivos hacia el sistema político y las percepciones negativas que suscita su funcionamiento real; por el contrario, la desafección política abarcaría tanto visiones desconfiadas y recelosas de las relaciones humanas, adquiridas en un estadio temprano del proceso de socialización, como percepciones contradictorias de la esfera política ${ }^{16}$. Por lo tanto, la

${ }^{16}$ Véase Citrin et al. (1975, 4-5). Morlino y Tarchi (1996, 47) también han distinguido dos formas de insatisfacción; mientras que la que ellos denominan insatisfacción pragmática se parece a nuestro concepto de descontento político, su insatisfacción ideológica, que llaman desafección, es diferente, ya que consideran que tiene consecuencias intrínsecamente peligrosas para la estabilidad del régimen a causa de su relación con valores culturales alternativos. 
desafección política, al contrario que la insatisfacción, tiende a ser más reacia al cambio y puede tener consecuencias más duraderas para la política democrática.

En esta sección sólo analizaremos dos de los muchos indicadores de desafección política existentes: la implicación psicológica de los ciudadanos en la política y su sentido de eficacia política. Como es bien sabido, la implicación psicológica en la política indica en qué medida los ciudadanos expresan un cierto interés o muestran alguna preocupación por la política y por los asuntos públicos. Los indicadores habituales de esta dimensión son el interés político subjetivo (definido por el grado de curiosidad que la política despierta en el ciudadano) (Van Deth, 1989, 281 y ss.) y la frecuencia con la que se discute de política (simbolizada por la expresión de interés en términos de un comportamiento que cristaliza en participación política informal) (Almond y Verba, 1963, 78 y ss.; Van Deth, 1991, y Topf, 1995). Ambos indicadores conforman un cuadro elemental de actitudes afectivas, percepciones de proximidad y sentimientos positivos dirigidos hacia la política. Y en ambos casos los datos españoles indican la existencia de una clara desafección que, además, se ha mantenido relativamente estable a lo largo del tiempo. En general, los españoles se diferencian poco de los ciudadanos de otros países occidentales en lo que se refiere al papel secundario que la política representa en sus vidas (Van Deth, 1989). Pero muestran, sin embargo, una mucho más acusada falta de interés en la política y una consiguiente menor frecuencia en las discusiones políticas. Como puede verse en el gráfico $2 \mathrm{a}$, los niveles de interés político y de las discusiones políticas han sido extremadamente bajos en España, a pesar de los enormes cambios políticos e institucionales que han tenido lugar en las dos últimas décadas ${ }^{17}$. El único aumento relativo tuvo lugar en los momentos iniciales de la transición, pero desde principios de los años ochenta alrededor del 40 por $100 \mathrm{de}$ los españoles declara que no tiene interés alguno en la política, y entre un 70 y un 80 por 100 afirma que su interés es escaso o nulo. Y tan notable como este desinterés generalizado resulta su firme continuidad, que contrasta además con la politización en forma de incremento del interés político observado en buena parte de las democracias europeas (Dalton, 1988, 22). Del mismo modo, y pese a algunas fluctuaciones temporales, el indicador de discusión política también ha permanecido bastante bajo. Como cabía esperar, esta falta de interés es mayor que en otros países de Europa occidental (Gabriel y Van Deth, 1995). Y estos indicadores de falta de implicación política son consistentes con otros aspectos del comportamiento político: por ejemplo, sólo el 5 por 100 de los españoles entrevistados en una

${ }_{17} \mathrm{Al}$ igual que en la mayoría de los estudios que utilizan estos indicadores (por ejemplo, Gabriel y Van Deth, 1995), aquí el interés por la política incluye a quienes están «muy» o «bastante» interesados en política, mientras que la frecuencia de las discusiones sólo incluye a quienes hablan de política «muy a menudo». 


\section{GRÁFICO 2a}

La desafección política en España: interés por la politica (1971-1996) y frecuencia de discusiones politicas (1983-1995)

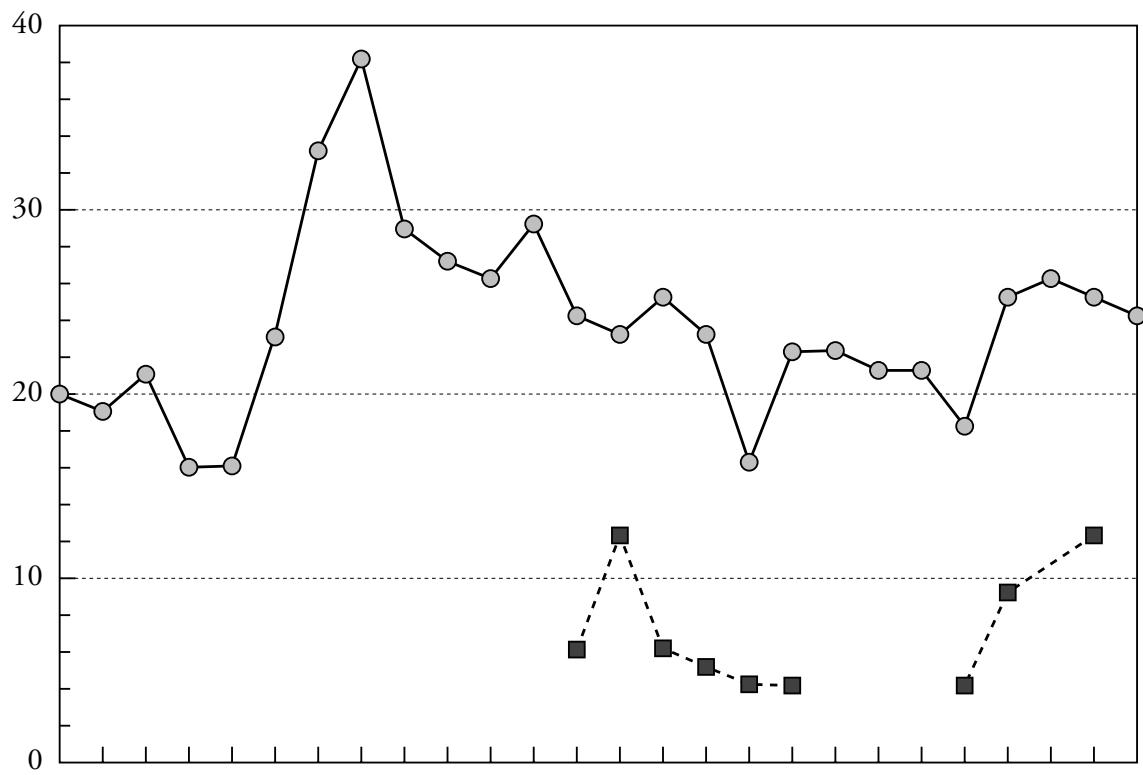

197172737475767778798081828384858687888990919293949596

$$
\text { -○- Interés - - D- Discusión }
$$

FueNTES: Monzón (1988, 109) para 1971-1977; Montero y Torcal (1990, 132) para 19781989, Banco de Datos del CIS y encuesta 1993 de DATA.

encuesta poselectoral de $1993^{18}$ afirmó que habían prestado «mucha atención» a la campaña electoral.

18 Esta encuesta, dirigida por José Ramón Montero, Richard Gunther, José María Maravall, Ludolfo Paramio, Francisco Llera y Francesc Pallarès, se llevó a cabo por DATA en la primavera de 1993 a una muestra representativa nacional de 1.400 españoles mayores de edad; se trataba de una encuesta de tipo panel, con una primera ola realizada antes del comienzo de la campaña electoral y una segunda (que logró una tasa de respuesta del 96 por 100) inmediatamente después de la jornada electoral. Los autores desean agradecer la generosa ayuda económica de la Comisión Interministerial de Ciencia y Tecnología (CICYT), que hizo posible la realización del estudio. 


\section{GRÁFICO 2b}

Desafección política en España: ineficacia politica interna y externa, 1978-1995*

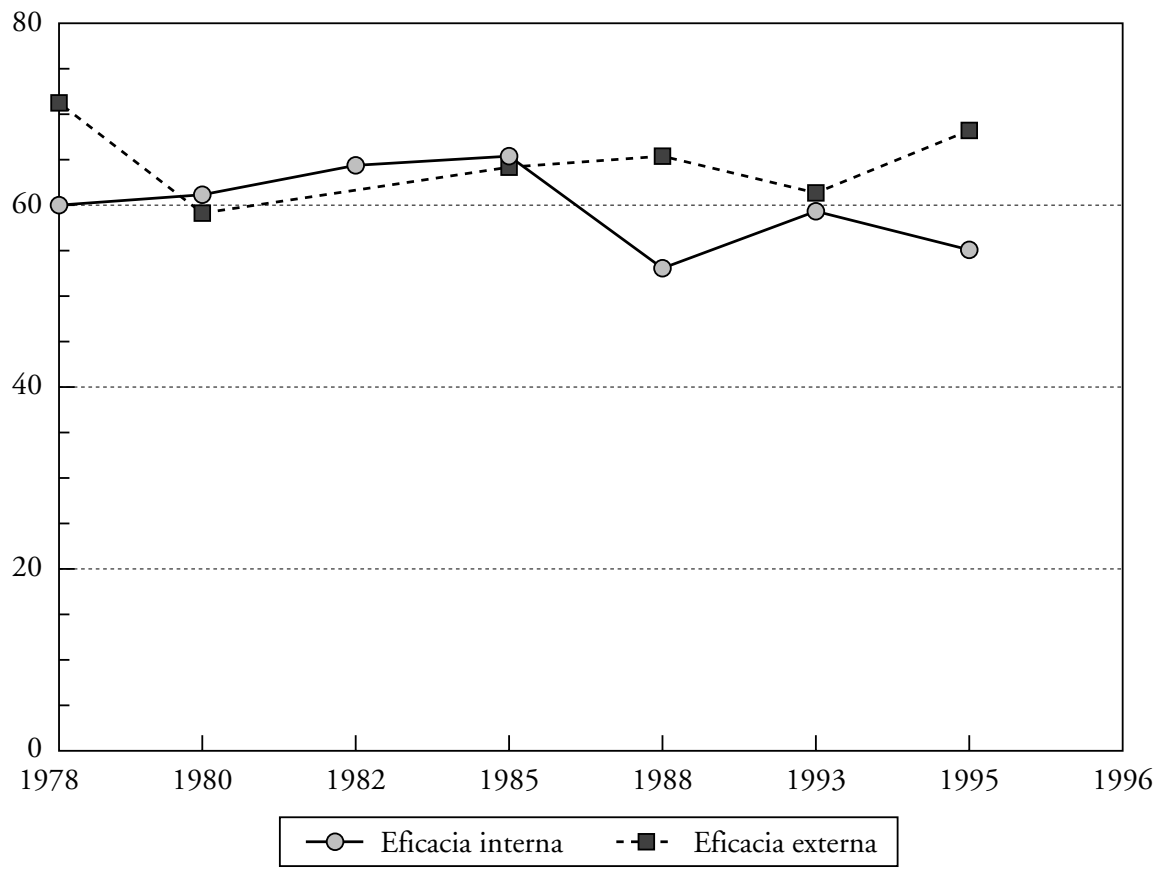

* Porcentaje de los que están de acuerdo con las afirmaciones correspondientes (véaase nota 19$)$.

Fuentes: Para 1978, 1980, 1985 y 1988, Banco de Datos del CIS; para 1979, 1982 y 1993 , encuestas de DATA, y para 1995, encuesta del Centro de Investigaciones para la Realidad Social (CIRES).

En líneas generales, el desinterés político se halla estrechamente vinculado a sentimientos de impotencia y confusión respecto a la política (Gunther, 1992, 15). Estos sentimientos se canalizan a través de una segunda faceta de la desafección, medida a través de la eficacia política del ciudadano. Es también sabido que este concepto integra una serie de actitudes básicas que expresan las percepciones que una persona tiene de sí misma y del sistema político. Desde hace ya tiempo es posible distinguir en la operacionalización empírica del concepto entre su faceta interna, que se refiere a la idea que el ciudadano tiene de su propia competencia (política) a la hora de entender la política y, en última instancia, de participar en ella, y una faceta externa, que se ocupa de la idea 
que tienen de la capacidad de respuesta de las autoridades e instituciones políticas a las demandas de los ciudadanos (Almond y Verba, 1963, 136 y ss.; Balch, 1974; Craig, Niemi y Silver, 1990, y Gabriel, 1995). Aunque ambos aspectos de la eficacia política suelen entenderse como parte de un concepto más amplio de insatisfacción política (Farah, Barnes y Heunks, 1979, 431-432), consideraremos por nuestra parte a ambas dimensiones como indicadores específicos de desafección. En el gráfico $2 \mathrm{~b}$ se presentan los datos españoles referidos a estos indicadores, que parecen apuntar a unos niveles de ineficacia llamativamente altos $^{19}$. A pesar de los habituales problemas de comparación, los pocos datos de que se dispone sugieren que los niveles españoles son más elevados que los de otros países europeos (Maravall, 1995, 291; Torcal, 1995, 150 y ss., y Gabriel, 1995) ${ }^{20}$. Entre la mitad y las dos terceras partes de los españoles mostraban su acuerdo con afirmaciones que denotaban sentimientos de ineficacia. Nuestra encuesta de 1993 incluía un indicador adicional de eficacia externa, por el que se solicitaba a los entrevistados que mostraran su acuerdo o desacuerdo con la afirmación de que "la gente como yo no tiene ninguna influencia en lo que hace el gobierno». La ineficacia política también resultó mayoritaria: el 64 por 100 de los españoles suscribía esa afirmación, mientras que sólo el 24 por 100 discrepaba de ella. Podría pensarse que estos altos niveles de ineficacia son simplemente una consecuencia de la juventud del sistema democrático, y que las manifestaciones de desafección evolucionarían con el tiempo a medida que los ciudadanos se familiarizaran con el funcionamiento de los mecanismos democráticos del sistema político. Pero no ha sido así. Los niveles de acuerdo con la afirmación relativa a que "los políticos no se preocupan» apenas ha variado en las numerosas encuestas realizadas desde 1978, mientras que sólo se ha producido una ligera reducción en la proporción de respuestas afirmativas al enunciado de que «la política es demasiado complicada» desde que comenzó a haber datos al respecto ${ }^{21}$.

Estas orientaciones parecen componer una característica estable, si no permanente, de la cultura política española ${ }^{22}$. Se reflejan en muchos otros indica-

19 La ineficacia interna se midió teniendo en cuenta si se suscribía la afirmación de que "la política es tan complicada que la gente como yo no puede entender lo que pasa»; y la externa, con la de que "los políticos no se preocupan mucho de lo que piensa la gente como yo".

20 Para dar sólo un ejemplo: en el Eurobarómetro, 45 (primavera de 1996), el 51 por 100 de los españoles declaraba que sus opiniones no tenían "ninguna influencia» en las decisiones adoptadas por su gobierno. Este porcentaje era el más alto de todos los países europeos, seguido por los de Bélgica (46 por 100), Reino Unido (43 por 100) y Francia (40 por 100). El promedio europeo era de 39 por 100 .

${ }^{21}$ Habría que señalar que durante algunos años los porcentajes disminuyeron debido al elevado número de no respuestas y al formato de las preguntas (ya que, en algunas, los encuestados podían optar por la categoría intermedia de «depende»). Pero, si se excluye a quienes no contestan, las cifras indican un sentimiento aún mayor y más estable de ineficacia política. Véase Torcal (1995, 86 y ss.).

22 Para una lúcida interpretación de algunas de las causas de esta estabilidad, véase Maravall (1995, 294 y ss.). 
dores que captan diferentes aspectos de este extrañamiento afectivo. Un análisis global de las diferentes actitudes hacia la política confirma la fuerza de esta desafección. Como puede comprobarse en la tabla 4, sólo alrededor de una tercera parte de los españoles selecciona sentimientos positivos; y, aunque únicamente una minoría expresa sentimientos nítidamente negativos, desde los años noventa la mayoría plantea su relación con la política en términos de inhibición, aburrimiento e indiferencia. Es cierto que estas orientaciones no son en absoluto privativas del caso español. Pero resulta probable que se hallen especialmente extendidas y que sean más intensas en España y, en general, en las nuevas democracias del sur de Europa (Morlino y Montero, 1995, 251-252; Sani, 1992).

\section{TABLA 4}

Sentimientos hacia la política, 1980-1996*

(en porcentajes)

\begin{tabular}{|c|c|c|c|c|c|c|c|}
\hline Sentimientos & 1980 & 1985 & 1989 & 1991 & 1993 & 1995 & 1996 \\
\hline \multicolumn{8}{|l|}{ Positivos } \\
\hline Apasionamiento .......................... & 1 & 1 & - & ** & ** & ** & 2 \\
\hline Entusiasmo $\ldots \ldots \ldots \ldots \ldots \ldots \ldots \ldots$ & $* *$ & 2 & 4 & 2 & 6 & 7 & 6 \\
\hline Compromiso ............................. & ** & 2 & 2 & 3 & 12 & $* *$ & 14 \\
\hline Interés & 24 & 24 & 19 & 18 & 26 & 26 & 30 \\
\hline \multicolumn{8}{|l|}{ Negativos } \\
\hline Irritación & 4 & 6 & 9 & 16 & 21 & 25 & 23 \\
\hline 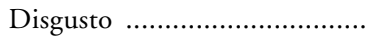 & $* *$ & 4 & - & $* *$ & $* *$ & $* *$ & 16 \\
\hline \multicolumn{8}{|l|}{ Desafectos } \\
\hline 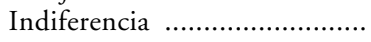 & 41 & 22 & 19 & 22 & 33 & 27 & 33 \\
\hline 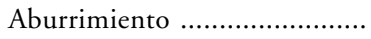 & 12 & 21 & 28 & 21 & 30 & 30 & 34 \\
\hline 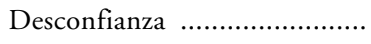 & 11 & 12 & 12 & 15 & 40 & 51 & 40 \\
\hline (N) & $(3.457)$ & $(2.498)$ & $(3.371)$ & $(2.471)$ & $(2.500)$ & $(3.983)$ & $(2.481)$ \\
\hline
\end{tabular}

* Desde 1980 a 1991, las cifras corresponden a la primera de las dos posibles respuestas que podía dar el entrevistado; desde 1993, respuestas múltiples. Las columnas pueden no sumar cien porque la no respuesta se ha excluido de la tabla.

** No se preguntó este año.

Podemos subrayar ahora que la evolución de las respuestas a estos indicadores no se ajusta a las pautas que antes examinamos de descontento político o de legitimidad democrática. A diferencia de los indicadores de insatisfacción, los de desafección no han fluctuado en paralelo a las condiciones generales de 
orden social, económico o político. En realidad, se han mantenido estables a pesar del desarrollo de los medios de comunicación desde finales de los años setenta y del aumento de los niveles de educación en los ochenta. Ni siquiera se vieron afectados por el desencanto de 1980-1981, ni por los mucho más positivos climas reinantes durante la transición a la democracia o la bonanza económica de finales de los ochenta. Tampoco acusaron las consecuencias de la alternancia de los partidos en el gobierno central, ni la extraordinaria descentralización del poder que supuso la creación del Estado de las autonomías. Y, a diferencia del apoyo a la democracia per se (que aumentó durante la transición y se estabilizó con la consolidación democrática, alrededor de 1982), los niveles de desafección se han mantenido prácticamente constantes a lo largo de las dos décadas transcurridas desde el fin de la dictadura. El sentimiento de eficacia política de los españoles no sólo es débil sino que, además, en los últimos años, no ha dado muestras de incremento alguno.

\section{LEGITIMIDAD, DESCONTENTO Y DESAFECCIÓN: TRES DIMENSIONES DIFERENTES}

En base principalmente a las diferentes tendencias de las dos últimas décadas, hemos mantenido como hipótesis que la legitimidad democrática, el descontento político y la desafección política son dimensiones conceptual y empíricamente distintas. Podemos ahora comprobar esta hipótesis. Nuestro primer paso consistirá en examinar si esos conceptos forman o no un conglomerado (un cluster, según la denominación usual) en el nivel individual, para analizar después cómo se distribuyen esas actitudes entre las diferentes generaciones de españoles. Por lo que hace al primer aspecto, contamos con un número suficiente de indicadores. Así, la ya citada encuesta poselectoral de 1993 incluía un indicador de legitimidad democrática (Legitim, tal y como aparece en las dos tablas siguientes, que expresaba la creencia de que "la democracia es el mejor sistema político para un país como el nuestro") ${ }^{23}$. También se recogían indicadores de descontento: evaluaciones de la situación económica (SitEcon) y política (SitPolit), el grado de satisfacción con «el funcionamiento de la democracia en España» (DemSat) y una valoración general de la actuación del gobierno (ActGob, que en el cuestionario era seguida por una batería de preguntas sobre la satisfacción respecto a las políticas de desempleo, educación, drogas, orden público, construcción de carreteras y desarrollo económico, así

${ }^{23}$ Hay que señalar que un análisis factorial anterior de los dos indicadores de legitimidad aquí utilizados (Legitim, cuyas distribuciones aparecen en la tabla 1, y las preferencias por un sistema democrático o por uno autoritario, que aparecen en tabla 2), junto al de los principales indicadores de descontento (Torcal, 1995, 109-114), han demostrado el alto grado de validez de ambos, de lo que se deduce que cualquiera de los dos puede utilizarse a efectos de comprobación de nuestra hipótesis. 
como asuntos exteriores y autonómicos). En la encuesta de 1993 se incluían asimismo cuatro indicadores de desafección: uno referido a implicación en política, consistente en el grado de interés por ella que el mismo encuestado confiesa tener (Interés); otro relativo a la eficacia interna, expresado por la creencia de que "la política es tan complicada" que el encuestado no puede entender lo que ocurre (CompPol); y otras dos cuestiones referidas a indicadores de eficacia externa, representados por las creencias de que a «los políticos no les preocupan" las preferencias de los ciudadanos (NoPreoc) y de que las personas como el entrevistado "no tienen ninguna influencia en lo que hace el gobierno» (NoInflu). Por último, la encuesta contenía tres indicadores de comportamientos que se relacionaban con las actitudes anteriores: el voto a favor o en contra del gobierno en las recientes elecciones generales (Voto 93), la frecuencia con la que el entrevistado discute de política (Discut) y su puntuación en un test simulado de información política $(\text { Info Test })^{24}$.

Hemos efectuado un análisis dimensional con las variables antes mencionadas $^{25}$. La tabla 5 refleja la estructura de las relaciones entre las variables. En ella se presentan, por encima de la línea punteada, las correlaciones entre todas las variables del primer conglomerado (que se refiere, evidentemente, a descontento político) y los coeficientes de saturación del primer factor que surgen del análisis factorial (rotación Varimax) ${ }^{26}$. El resto de las variables se presenta por debajo de la línea punteada, junto a sus correlaciones con los de descontento político y sus coeficientes de saturación respecto al primer factor. Como puede comprobarse, todos los indicadores de descontento político antes analizados pertenecen al mismo conglomerado: tanto los coeficientes de saturación como las correlaciones entre las variables son bastante fuertes. Este con-

${ }^{24}$ La puntuación en este test de información se basaba en la capacidad del entrevistado para señalar el nombre correcto del ministro de Hacienda, del secretario general de Comisiones Obreras, del presidente del Congreso de Diputados y del presidente de la Confederación Española de Organizaciones Empresariales (CEOE).

${ }_{25}$ Para análisis dimensionales similares pueden verse los trabajos de Craig, Niemi y Silver (1990) para el caso de Estados Unidos y mediante un estudio piloto de 1987 del National Election Study, que incluía más de 35 indicadores de eficacia y confianza; Kaase (1994) para cinco países de Europa oriental incluidos en el Eurobarometer East Survey de 1992, que tenía un número limitado de variables referidas sólo a la satisfacción económica, la satisfacción política y la participación política; Kornberg y Clarke (1992, 114 y ss.) para el caso canadiense, y, para el espanool, Torcal (1995, 216 y ss.), Maravall (1995, 278 y ss.) y McDonough, Barnes y López Pina (1986 y 1994).

${ }^{26}$ Los resultados del análisis factorial que aparecen en las tablas 5 y 6 se obtuvieron mediante una rotación Varimax en la que el número de factores se limitó a dos. En análisis anteriores aparecía también un tercer factor más débil (que se componía del indicador de legitimidad democrática y de dos medidas de eficacia externa). Sin embargo, como las correlaciones entre legitimidad democrática y esas dos medidas eran extraordinariamente bajas $(0,04$ y 0,11$)$, era evidente que el tercer factor surgía por defecto a consecuencia de la relativa debilidad de la relación entre las dos medidas de eficacia externa y las otras del conglomerado de desafección, al mismo tiempo que parecía claro que el ítem de legitimidad democrática no encajaba con ninguno de los de descontento o desafección y, por tanto, carecía de «cabida» en otro «sitio». 
glomerado incluye a las diferentes facetas de la dimensión del descontento político. En primer lugar, el grado de satisfacción con el funcionamiento de la democracia en España y las valoraciones sobre la situación económica y la política están relacionados. En segundo lugar, como se apuntaba en nuestra hipótesis, el sentimiento de satisfacción (con cualquier objeto político) tiene un carácter netamente partidista y está asimismo relacionado con las valoraciones respecto a la actuación del gobierno. Finalmente, y como también se señalaba en nuestra hipótesis, la principal consecuencia de estos indicadores de satisfacción en el ámbito del comportamiento es la tendencia a votar contra el partido en el gobierno.

\section{TABLA 5}

El conglomerado del descontento político: análisis factorial y matriz de correlaciones entre las variables

\begin{tabular}{|c|c|c|c|c|c|c|}
\hline & SitEcon & SitPolit & DemSat & Voto93 & ActGob & $\begin{array}{c}\text { Coeficiente } \\
\text { de } \\
\text { saturación }\end{array}$ \\
\hline SitEcon & - & & & & & 0,685 \\
\hline SitPolit .............................. & 0,49 & - & & & & 0,706 \\
\hline 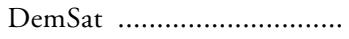 & 0,31 & 0,36 & - & & & 0,670 \\
\hline 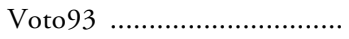 & 0,30 & 0,32 & 0,30 & - & & 0,678 \\
\hline 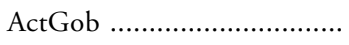 & 0,45 & 0,46 & 0,50 & 0,57 & - & 0,827 \\
\hline 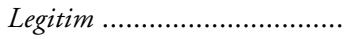 & 0,05 & 0,11 & 0,13 & $-0,03$ & 0,11 & 0,113 \\
\hline 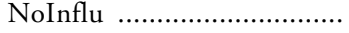 & $-0,06$ & $-0,06$ & $-0,08$ & $-0,10$ & $-0,08$ & $-0,201$ \\
\hline NoPreoc & $-0,16$ & $-0,15$ & $-0,22$ & $-0,17$ & $-0,20$ & $-0,381$ \\
\hline 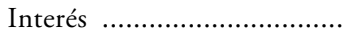 & $-0,03$ & 0,04 & 0,07 & $-0,09$ & $-0,04$ & $-0,005$ \\
\hline 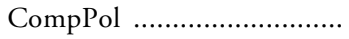 & 0,00 & $-0,01$ & $-0,05$ & 0,04 & 0,01 & 0,046 \\
\hline 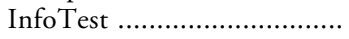 & 0,06 & $-0,01$ & $-0,05$ & 0,14 & 0,08 & 0,074 \\
\hline 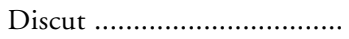 & 0,07 & $-0,05$ & 0,00 & $-0,12$ & $-0,09$ & $-0,104$ \\
\hline
\end{tabular}

Fuente: Encuesta DATA, 1993.

Por otra parte, es también evidente que la variable de legitimidad democrática (Legitim) no pertenece a este conglomerado del descontento político, y que tampoco se correlaciona de forma considerable o consistente con ninguno de sus indicadores. Esto confirma que ambas dimensiones actitudinales son distintas y que, además, son relativamente autónomas ${ }^{27}$. Resulta asimismo

27 Craig, Niemi y Silver $(1990,306)$ presentan una conclusión similar, al igual que lo hacen, en el caso español, utilizando diferentes técnicas y/o datos, Maravall (1995, 279) y Morlino y Montero (1995). 
claro que los diversos indicadores de desafección pertenecen a una tercera dimensión diferente, lo cual confirma por su lado su separación con respecto a las principales facetas del descontento político. La única excepción parcial es la eficacia externa (NoPreoc), que se relaciona en mayor medida con los indicadores de insatisfacción política que el resto (incluyendo los de eficacia interna). Se trata de una excepción no muy sorprendente, ya que refleja la propensión a evaluar a las autoridades políticas en términos relacionados con la apertura y la receptividad del sistema político ${ }^{28}$. Por lo tanto, resulta esperable que los encuestados que no estén satisfechos con la situación económica y/o política culpen al gobierno (evaluando negativamente su actuación y eventualmente votando contra los candidatos del partido del gobierno) y que, a la larga, tiendan a relacionar las actuaciones negativas del gobierno con la creencia de que a los políticos no les preocupa lo que piensa la gente. De este modo, cabría concluir que las habituales equiparaciones entre satisfacción con el sistema y legitimidad, o sus contrarias, carecen de fundamento.

Los datos presentados en la tabla 6 evidencian que existe un conglomerado bien definido de indicadores de desafección politica, aunque sea con menor nitidez que en el caso anterior. En realidad, y como cabía esperar, los coeficientes de saturación de las variables que expresan una implicación psicológica en la política (Interés y Discut) están entre los más altos. Algo similar puede decirse de las concepciones sobre la complicación e incomprensibilidad de la política $(C o m p P o l)$, comprendidas en una variable que representa la dimensión de la eficacia interna. El conglomerado de todas estas variables señala acertadamente los elementos de desapego, alejamiento y desconfianza que forman parte del concepto más general de desafección política. Por el contrario, los coeficientes de saturación de los indicadores de eficacia externa (NoPreoc y NoInflu) son más bajos que los de otras variables. No obstante, parecen estar asociados a los restantes indicadores de desafección, y en la dirección esperada. Por ejemplo, los coeficientes negativos de eficacia interna son mucho más fuertes con las variables de implicación psicológica en política que los de eficacia externa; y los indicadores de eficacia interna y externa presentan algunas correlaciones positivas significativas, denotando probablemente cómo las valoraciones que hacen los ciudadanos de su eficacia política configuran la visión que tienen del papel que ellos mismos pueden desempeñar en política o sobre la receptividad del sistema político a sus demandas ${ }^{29}$. Aún más importante es que ni la legitimidad democrática ni el descontento político parezcan estar relacionados de modo relevante con el conglomerado de variables de desafección política. De nuevo, este hecho cuestiona la suposición de que las dimensiones de desafección puedan considerarse indicadores de ineficacia del sistema o de insatisfacción política. Además de los datos presentados anteriormente sobre la diferente evolución temporal de estas actitudes, este análisis factorial ha permitido una

28 Véase también Craig, Niemi y Silver (1990, 306).

29 Véase, para argumentos similares, Craig, Niemi y Silver (1990, 305). 
primera comprobación de nuestra hipótesis: tras su realización, parece evidente que las actitudes de legitimidad, descontento y desafección son diferentes unas de otras tanto en términos conceptuales como empíricos ${ }^{30}$.

\section{TABLA 6}

El conglomerado de la desafección política: análisis factorial y matriz de correlaciones entre las variables

\begin{tabular}{|c|c|c|c|c|c|c|c|}
\hline & Interés & CompPol & Info Test & Discut & NoInflu & NoPreoc & $\begin{array}{c}\text { Coeficiente } \\
\text { de } \\
\text { saturación }\end{array}$ \\
\hline Interés …............... & - & & & & & & $-0,799$ \\
\hline CompPol ........... & $-0,36$ & - & & & & & 0,635 \\
\hline InfoTest ............. & $-0,38$ & $-0,26$ & - & & & & 0,639 \\
\hline Discut .................. & 0,58 & $-0,29$ & 0,34 & - & & & $-0,750$ \\
\hline NoInflu .............. & $-0,10$ & 0,17 & 0,09 & $-0,08$ & - & & 0,298 \\
\hline NoPreoc .................. & $-0,17$ & 0,25 & 0,06 & $-0,12$ & 0,28 & - & 0,375 \\
\hline Legitim ................. & 0,09 & 0,02 & $-0,14$ & 0,08 & 0,04 & 0,11 & $-0,125$ \\
\hline SitEcon...$\ldots \ldots \ldots \ldots$ & $-0,03$ & 0,00 & 0,06 & 0,07 & $-0,06$ & $-0,16$ & 0,056 \\
\hline SitPolit ..................... & 0,04 & $-0,01$ & $-0,01$ & $-0,05$ & $-0,06$ & $-0,15$ & $-0,019$ \\
\hline DemSat .................... & 0,07 & $-0,05$ & $-0,05$ & 0,00 & $-0,08$ & $-0,22$ & $-0,113$ \\
\hline Voto93 .................. & $-0,09$ & 0,04 & 0,14 & $-0,12$ & $-0,10$ & $-0,17$ & 0,158 \\
\hline 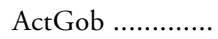 & $-0,04$ & 0,01 & 0,08 & $-0,09$ & $-0,08$ & $-0,20$ & 0,076 \\
\hline
\end{tabular}

Fuente: Encuesta DATA, 1993.

\section{PAUTAS INTERGENERACIONALES DE CONTINUIDAD Y CAMBIO}

Una segunda forma de comprobar la diferencia entre legitimidad democrática, descontento político y desafección política consiste en examinar sus respectivas pautas de cambio y continuidad en distintas cohortes de edad. Además, si comparamos estas pautas en diferentes generaciones, podemos plantear algunas hipótesis adicionales sobre los orígenes de estas orientaciones, así como

${ }^{30}$ Los resultados del análisis factorial llevado a cabo por Kaase $(1994,269)$ en cinco países de Europa oriental dieron como resultado, en cuatro de ellos, dos factores: la implicación política (el más pequeño de los dos) y la satisfacción/insatisfacción (el más importante, y en el que los coeficientes de saturación de todas las variables económicas y políticas eran igualmente altos). Sin embargo, el autor, al no poder incluir una variable que midiera la legitimidad democrática, tampoco pudo describir las interacciones recíprocas, mucho más interesantes, entre legitimidad, descontento y desafección. 
sobre los factores que han tenido incidencia en ellos a lo largo de la vida de los ciudadanos. Aquí haremos un análisis longitudinal de los datos referidos a esas actitudes (utilizando datos de encuestas realizadas a lo largo del período que nos interesa) para revelar la existencia de diferentes generaciones políticas en España. El concepto de generación política va mucho más allá de la biología: está basado en la idea de que acontecimientos políticos señalados marcan a las distintas generaciones, confiriéndoles unas pautas peculiares y duraderas de actitudes y comportamientos políticos (Mannheim, 1952, 276 y ss.). Como en estudios similares llevados a cabo en otros países ${ }^{31}$, nuestro punto de partida básico es que cada generación de españoles se ha visto marcada por los acontecimientos sociales y políticos que tuvieron lugar durante la época más significativa de su socialización, y que dichos acontecimientos han seguido influyendo en sus actitudes y en su comportamiento a lo largo de su vida. Otros análisis más matizados han demostrado que el impacto real de un acontecimiento histórico sobre una generación no procede de las experiencias personales de quienes lo han vivido directamente, sino de cómo ese acontecimiento es reconstruido, interpretado y recreado en la memoria colectiva (Schuman y Scott, 1989).

Como es bien sabido, un diseño longitudinal de cohortes puede detectar tres tipos de efectos relevantes para la explicación de la estabilidad y del cambio actitudinal. En primer lugar, está el efecto cohorte: algunas actitudes presentan diferencias generacionales persistentes y duraderas que apenas cambian con acontecimientos políticos concretos. En segundo lugar, hay un efecto período: algunas actitudes cambian en todas las generaciones como consecuencia de un acontecimiento que las afecta a todas ellas, pero sin que pueda observarse una pauta clara o consistente. El tercero es el efecto ciclo vital: algunas actitudes cambian simplemente con la edad. Hemos realizado un análisis de cohortes a las actitudes de legitimidad, descontento y desafección, examinando cómo han cambiado durante los últimos quince años en seis generaciones de españoles ${ }^{32}$. Podremos así comprobar que la legitimidad

${ }^{31}$ Para un análisis de cohortes similar en Alemania desde los años cuarenta, véase Weil (1987).

${ }^{32}$ Las seis generaciones fueron definidas en relación a los acontecimientos históricos más significados del siglo XX. La cohorte mayor comprende a los nacidos antes de 1922, es decir, a los que llegaron a la edad adulta al final de la Monarquía, durante la Segunda República o la Guerra Civil. La cohorte siguiente (cohorte 5), conocida como la generación de la autarquía, se compone de las personas nacidas entre 1923 y 1937, que llegaron a la madurez política durante los difíciles años de la depresión económica de la posguerra. La cuarta generación (cohorte 4) es la del despegue económico, e incluye a los nacidos entre 1938 y 1952, que llegaron a la mayoría de edad cuando el control económico pasó de los falangistas a los tecnócratas que pusieron en práctica los Planes de estabilización y el desarrollo de los años sesenta. La tercera generación (cohorte 3) es la de la liberalización del régimen, esto es, la de quienes nacieron entre 1953 y 1962 y se hicieron políticamente conscientes durante la liberalización y la crisis del régimen. La segunda generación (cohorte 2) es la de la transición y comprende a las personas que nacieron entre 1963 y 1967 y que alcanzaron su madurez política en la transición al nuevo sistema democrático y en su consolidación. Finalmente, la cohorte más joven es la de quienes han nacido después de 1968 y que, en consecuencia, sólo han conocido la democracia. 
y la desafección muestran un efecto cohorte de forma clara y consistente, es decir, que se han mantenido estables con el desarrollo de la edad y que las únicas diferencias apreciables entre ellas se refieren a las distintas pautas de continuidad en distintas generaciones de españoles. Por el contrario, en las actitudes que se relacionan con las evaluaciones de la situación económica y política hay un efecto período, lo cual les otorga una mayor inestabilidad.

Veamos, en primer lugar, qué ocurre con la dimensión de la legitimidad. Entre las generaciones seleccionadas, la comparación de las preferencias por un sistema democrático muestra que existe un claro efecto cohorte ${ }^{33}$. Como puede apreciarse en el gráfico 3, las diferencias de cada generación en el apoyo incondicional a la democracia son estables y significativas; esto ocurre incluso entre la tercera cohorte (la de los nacidos entre 1953 y 1962, que alcanzaron la mayoría de edad inmediatamente después de la transición a la democracia) y la cuarta (la de los nacidos entre 1938 y 1952, que experimentaron un intenso crecimiento económico durante sus años de formación). Cuanto más joven es la cohorte, mayor es el apoyo a la democracia, aunque las dos más jóvenes no se diferencian a este respecto ${ }^{34}$. Cabe observar un cambio intergeneracional en el grado de apoyo al nuevo régimen democrático entre la tercera y la cuarta generación (que llegó a la madurez durante los años de la modernización económica y social y la subsiguiente liberalización y expansión educativa de los años sesenta y setenta), pero también se aprecia esta tendencia entre la cuarta y la quinta generación. Estos datos revelan las consecuencias que tienen las diferentes circunstancias en las que estas generaciones de españoles fueron socializadas y adquirieron sus actitudes políticas básicas. También reflejan la existencia de memorias colectivas diferenciadas del fracaso de la Segunda República, de la Guerra Civil y de las diferentes fases del régimen autoritario. Como ha demostrado Aguilar (1996), estas memorias fueron «recreadas» durante el franquismo por las distintas generaciones de españoles, produciendo una especie de compromiso difuso para no repetir la aún reciente tragedia colectiva de la guerra, las situaciones de conflicto crónico y las manifestaciones de intolerancia. De este modo, y a pesar de algunas diferencias generacionales, la democracia gozaba ya en los primeros años setenta de un apoyo considerable; un apoyo que reflejaba la existencia de una serie de actitudes favorables a ella incluso antes de que comenzara la transición (Montero y Gunther, 1994; Maravall, 1995, 275). Estas actitudes se vieron reforzadas posteriormente por el indiscutible éxito de la transición democrática, que generó un efecto luna de miel en los primeros años del nuevo sistema político (Linz y Stepan, 1996, 101).

A pesar del predominio de las diferencias intergeneracionales, deben desta-

${ }_{33}$ Para una mayor claridad, el gráfico 3 no muestra la línea de la cohorte más joven, que es prácticamente igual a la de la cohorte 2 .

${ }^{34} \mathrm{El}$ apoyo a un régimen autoritario, la otra alternativa por la que podían optar los encuestados, presenta una pauta opuesta: cuanto más joven es la generación, menos apoyo muestra por esta opción. Véanse Morlino y Montero (1995, 136-137) y Montero (1993, 149-152). 


\section{GRÁFICO 3}

Legitimidad por cohortes, 1980-1994

(porcentaje de quienes declaran que la democracia es preferible a cualquier otra forma de gobierno)

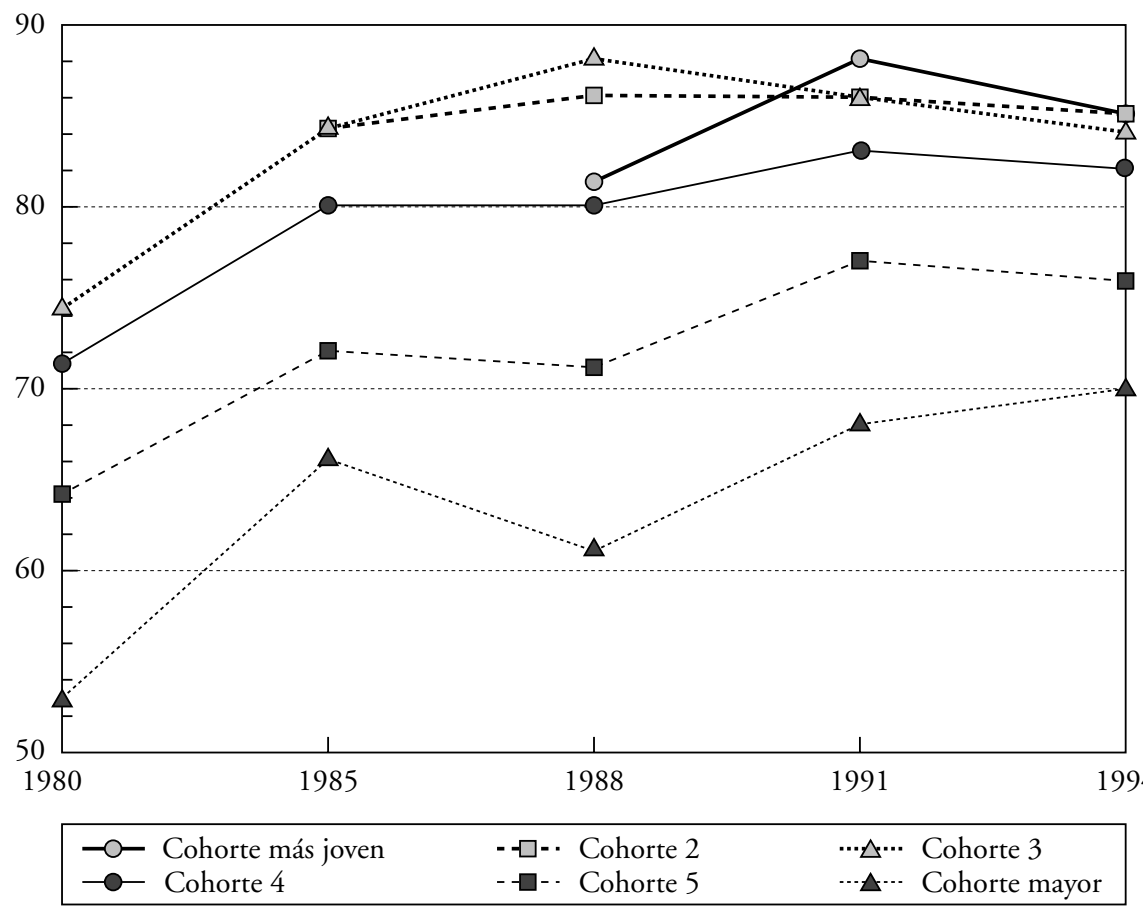

Fuente: Banco de Datos del CIS.

carse dos aspectos más del gráfico 3. El primero es el importante efecto período que se observa en el incremento de la legitimidad entre 1980 y 1985, que se ha mantenido estable desde entonces; el segundo es la convergencia progresiva entre generaciones jóvenes y viejas en lo que respecta al grado de apoyo al régimen, según puede apreciarse en las pequeñas diferencias que se registran en 1994. Estos aspectos apuntan a procesos de resocialización actitudinal adulta y/o de aprendizaje político (Bermeo, 1992, 274; Aguilar, 1996, 355 y ss.). Los efectos período indican que, a medida que se consolidaba el nuevo régimen entre 1980 y 1985, los españoles debieron conocer nuevos cambios actitudinales, que en cualquier caso tuvieron consecuencias positivas en sus orientaciones 
hacia la democracia (López Pintor, 1987, 1006-1007; Maravall, 1995, 263). Estos cambios quedaron reflejados en el aumento de la legitimidad otorgada al nuevo régimen desde 1980; un aumento que, pese a la continuidad de las diferencias entre las diversas cohortes, afectó a todas por igual. Del mismo modo, la leve pero progresiva convergencia de todas las líneas en años sucesivos revela que, una vez estabilizadas las actitudes de apoyo al régimen, su aceptación ha aumentado lentamente entre las generaciones más mayores. Esto sugiere que el ethos democrático ha ido claramente ganando terreno entre aquellas generaciones cuya socialización tuvo lugar casi por completo bajo el régimen autoritario, y que por lo tanto aceptaron el nuevo régimen democrático en términos relativamente menores. Aunque sigue predominando el efecto cohorte, el efecto período sugiere que el paso del tiempo, con sus procesos de aclimatación y socialización, también favorece el apoyo a la democracia sobre el de otros regímenes alternativos. En este sentido, el caso español confirma las conclusiones de los estudios comparativos que han subrayado las consecuencias positivas de una combinación de dos factores para la adquisición de altos grados de legitimidad: un incremento en el apoyo a la democracia después de un corto período de funcionamiento del nuevo régimen y, sobre todo, la presencia, antes del cambio de régimen, de actitudes favorables hacia la democracia que no procedan del proceso de modernización, sino de la comparación histórica que cada generación hace de los regímenes en los que ha vivido, así como de la experiencia de otros países que han disfrutado de sistemas democráticos y que sirven como "grupos de referencia» (Weil, 1993, 198).

La dimensión del descontento político, en segundo lugar, arroja resultados distintos a los de la legitimidad. De hecho, un análisis de cohortes del grado de satisfacción de los españoles con el funcionamiento de la democracia presenta una pauta muy diferente. El gráfico 4, que incluye el porcentaje de ciudadanos de cada cohorte que afirma que "la democracia funciona bien» o "aceptablemente bien", revela la ausencia de un efecto cohorte: apenas existen diferencias entre las generaciones y, cuando las hay, se produce una intersección entre las líneas, es decir, que oscilan de un año a otro sin que pueda identificarse una pauta común. Existe también un claro efecto período entre 1991 y 1994, coincidiendo con los fenómenos de corrupción y escándalos políticos, como puede apreciarse en el descenso de la satisfacción respecto al funcionamiento de la democracia en todas las cohortes. De ahí que afecte por igual a todos los ciudadanos, independientemente de la generación a la que pertenecen, y que produzca una drástica caída de la satisfacción política ${ }^{35}$. Estos datos no sólo reflejan una vez más la autonomía actitudinal de la legitimidad y de la eficacia, sino que señalan también su peculiar naturaleza. Mientras que los niveles de

35 De hecho, la única generación que destaca por su evaluación positiva es la mayor, una aparente anomalía que se debe probablemente al apoyo de sus integrantes a las mejoras en las pensiones y en la seguridad social introducidas por los Gobiernos socialistas. 
legitimidad se diferencian para cada generación y tienden a permanecer estables durante todo el ciclo vital del individuo, la satisfacción con el rendimiento de la democracia es inestable y depende mucho más de las actividades de los gobiernos. El básicamente incondicional apoyo de los españoles a la democracia no se ha visto así afectado por las difíciles condiciones económicas impuestas por la recesión y el desempleo, ni por las dificultades creadas por situaciones políticas complejas ni por el impacto negativo derivado de los casos de corrupción o de escándalos políticos.

\section{GRÁFICO 4}

Eficacia del sistema por cohortes, 1985-1994

(porcentaje de quienes declaran que la democracia funciona bien o que tiene muchos defectos pero funciona razonablemente bien)

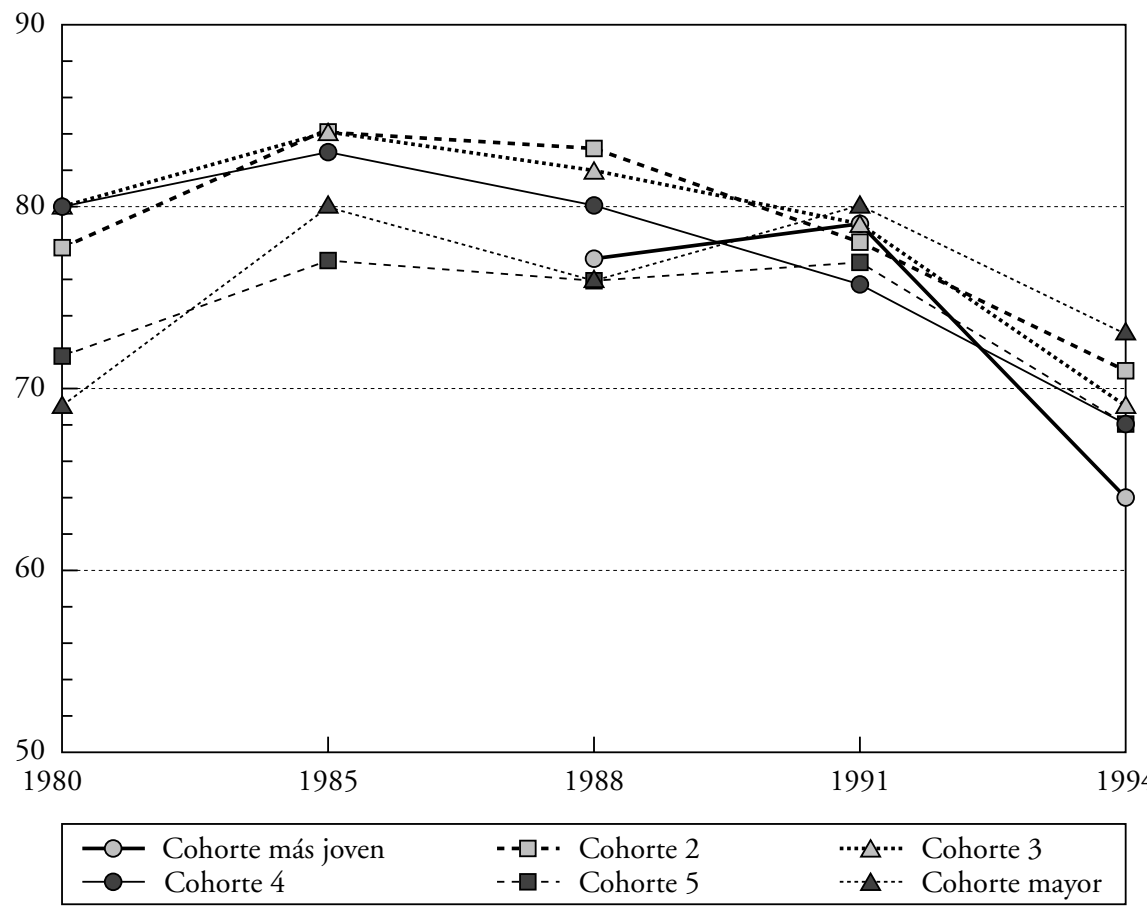

Fuente: Banco de Datos del CIS. 
Queda, finalmente, la dimensión de la desafección política. En principio, la medida de la desafección arroja un efecto generacional. Pero, al contrario de lo observado en el caso de la legitimidad, sus indicadores muestran pocas diferencias intergeneracionales. Dicho de otro modo, las actitudes que se relacionan con la desafección parecen transmitirse de una generación a otra sin apenas cambios. Esta continuidad es ciertamente notable si se tienen en cuenta las transformaciones sociales y económicas de los últimos treinta años, y los cambios políticos de los últimos veinte. Para ilustrar este punto hemos elegido la eficacia política externa de entre los indicadores de desafección política anteriormente analizados. El gráfico 5 muestra la evolución por cohortes de la proporción de españoles que está en desacuerdo con la afirmación relativa a que «los políticos no se preocupan mucho de lo que piensa la gente como yo». Como es fácilmente apreciable, las diferencias entre las generaciones resultan escasas. La percepción de la eficacia externa tampoco cambia en función del ciclo vital. En otras palabras, no mejora a medida que se envejece: nótese que no se producen incrementos progresivos en ninguna de las líneas. Al mismo tiempo, apenas crece de una generación a la siguiente: hay poca distancia entre las líneas. Existen algunos efectos período, pero su bajo valor porcentual indica que son poco significativos. Por lo tanto, parece que los cambios sociales, políticos y económicos no tienen incidencia en la valoración de la eficacia externa realizada por las distintas generaciones de españoles durante los últimos quince años. Estos resultados confirman la hipótesis de que la eficacia política y otras actitudes similares de desafección proceden de un largo proceso de «acumulación cultural» (Almond y Verba, 1963, 213 y ss. y 279; Putnam, 1993, 152162): se mantienen estables entre las distintas generaciones, al margen de los cambios sistémicos, y son difícilmente modificables. Estos datos corroboran nuevamente una afirmación ya conocida en la literatura comparada: los sentimientos de eficacia política se caracterizan por una notable continuidad intergeneracional ${ }^{36}$.

Las diferencias que hemos examinado contienen algunas implicaciones destacables. En primer lugar, las actitudes que miden la desafección política presentan una considerable estabilidad a través de las generaciones, lo cual contrasta con las extraordinarias diferencias intergeneracionales que se observan en todos los indicadores relativos a la modernización, la educación y la religiosidad (Torcal, 1995). En segundo lugar, el descontento político no presenta efecto cohorte alguno: varía de una generación política a otra sin que parezca seguir una pauta definida. En tercer lugar, y al contrario de la pauta correspondiente a la desafección política, la legitimidad democrática ha sufrido importantes cambios intergeneracionales. Pero este aumento, que ha sido similar en todas las generaciones, no es una mera consecuencia de los procesos de modernización o de desarrollo económico. Si así fuera, las diferencias en el

${ }^{36}$ Aunque este resultado no parece deberse a la transmisión actitudinal de padres a hijos; véanse Jennings y Niemi (1981, 203-205), Dalton (1980, 412-431) y Abramson (1983, 146-147). 


\section{GRÁFICO 5}

Eficacia politica externa por cohortes, 1980-1995

(porcentaje de quienes no están de acuerdo con la afirmación de que «los políticos no se preocupan mucho de lo que piensa la gente como yo»)

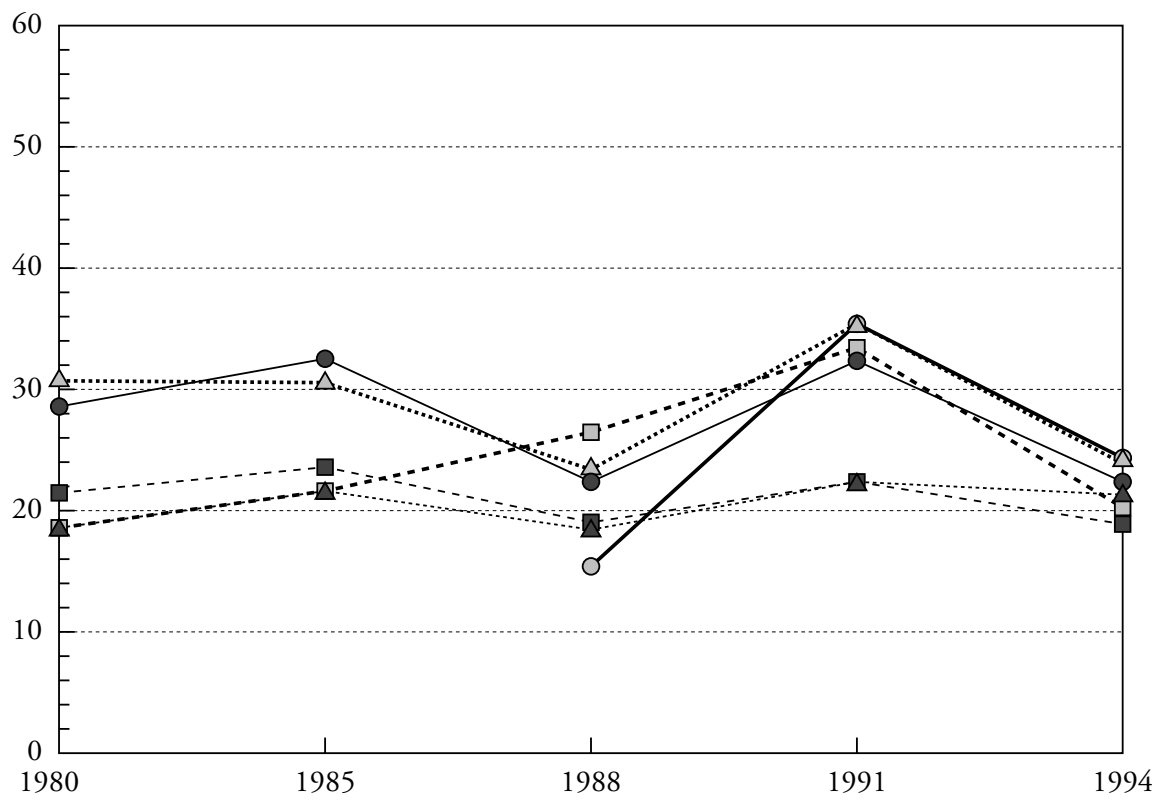

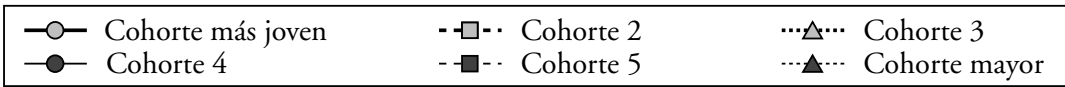

Fuente: Banco de Datos del CIS.

apoyo al régimen habrían sido más acusadas en las generaciones que sufrieron en mayor medida las transformaciones económicas y sociales de los años sesenta; un resultado que no llegó a producirse, ya que se mantienen las mismas diferencias en todas las generaciones. Por lo tanto, la modernización que tuvo lugar en los años sesenta y setenta no cambió per se las pautas actitudinales de los españoles en lo que hace a la democracia. En todo caso, puede que haya favorecido la creación de una base común sobre la que se asentaran los cambios actitudinales registrados en todas las generaciones una vez ya comenzada la transición a la democracia. Sin embargo, las similitudes y diferencias procedentes de experiencias previas a la edad adulta han continuado siendo igualmente pronunciadas. El cambio actitudinal, cuando tiene lugar, parece deberse 
a la presencia de diversas memorias colectivas que condicionan la interpretación del presente y que cada generación política reinterpreta de forma diferente.

\section{OBSERVACIONES FINALES}

La cultura política es un fenómeno multidimensional. Pese a ello, los vínculos existentes entre sus distintas dimensiones no han recibido la atención que merecen. La mayoría de los estudios sobre este tema ha señalado, siguiendo a Almond y Verba (1963) implícita o explícitamente, que los diferentes conjuntos de actitudes deben seguir pautas coherentes y consistentes. Esta premisa ha tenido importantes consecuencias para nuestra comprensión de la cultura política en general, y más particularmente de las actitudes hacia el sistema político. Por ejemplo, la literatura sobre la cultura política de las viejas democracias se ocupa de numerosos casos en los que se interpreta que cualquier signo de insatisfacción con la democracia puede conducir a una crisis de la legitimidad democrática. De modo similar, la supuesta coherencia entre las percepciones de apoyo a la democracia y las evaluaciones de su rendimiento ha sido atribuida a diversas dimensiones del concepto de satisfacción con la democracia, pasando así por alto la distinta naturaleza de los objetos políticos que se integran en dicho concepto y la diversidad existente en las orientaciones básicas de distintos tipos de ciudadanos. Los datos que hemos analizado sobre las actitudes hacia la democracia en España parecen indicar que las relaciones son bastante más complejas. El análisis de la evolución de la legitimidad democrática, el descontento político y la desafección política entre los españoles durante los últimos veinte años ha producido, según creemos, varias conclusiones teóricas significativas.

En primer lugar, y pese al todavía reciente proceso de transición democrática española, el respaldo que el nuevo régimen recibía ya en los años ochenta era tan amplio como el de otros países de Europa occidental. Además, ese apoyo ha disfrutado de una llamativa estabilidad durante las dos décadas posteriores a pesar de las difíciles y a veces turbulentas circunstancias que rodearon a la transición política (con altos niveles de violencia política, un fallido golpe de Estado y crisis económicas sucesivas que incrementaron el índice de desempleo al 20 por 100 de la población activa).

En segundo lugar, la utilización de diversos tipos de indicadores empíricos nos ha permitido distinguir entre legitimidad democrática, por un lado, y eficacia del sistema y satisfacción con el funcionamiento de la democracia, por otro. Al contrario que en la mayoría de los países europeos, en los que se carece de datos muestrales adecuados para diferenciar esas dos dimensiones (véase Kaase y Newton, 1995, 168), las encuestas españolas hacen posible el análisis de sus relaciones y de su evolución. Y lo hacen, además, con la cualificación añadida de unas condiciones a veces muy difíciles, en las que se combinaban la 
incertidumbre de un proceso de transición, la difícil situación económica a principios de los años noventa (tanto en España como en la mayoría de las democracias occidentales) y casos llamativos de corrupción y escándalos políticos. Aunque ambas corrientes de insatisfacción tuvieron consecuencias políticas importantes (entre las que destacaron el declive de los apoyos electorales de los partidos en el gobierno y la reestructuración del sistema de partidos), no han producido un descenso significativo ni persistente del apoyo a la democracia, ni tampoco incremento electoral alguno a los partidos antisistema.

En tercer lugar, la desafección política, medida a través de la implicación psicológica en política y de la eficacia política tanto externa como interna, ha resultado también ser una dimensión actitudinal independiente. Entre los españoles se registra un alto grado de desafección política, que se ha mantenido estable durante los últimos veinte años pese a los extraordinarios cambios ocurridos en los ámbitos sociales, educativos, económicos y, sobre todo, políticos. Además, y en contra de lo que algunos autores (Muller y Selignon, 1994) han señalado respecto a la relación causal entre las actitudes cívicas y la democracia, el caso español indica con claridad que la desafección política no siempre disminuye con el mero paso del tiempo, ni siquiera cuando en ese tiempo transcurre el establecimiento, la consolidación y la institucionalización de un régimen democrático. La desafección política parece constituir un fenónemo cultural que presenta una notable estabilidad.

Finalmente, el análisis factorial ha aportado evidencia significativa sobre la diferenciación teórica y empírica de las actitudes de legitimidad democrática, descontento político y desafección política. Y el análisis de cohortes ha permitido, además, comprobar sus respectivos niveles de cambio y continuidad generacionales. Los resultados subrayan la distinta naturaleza de cada una de estas actitudes y confirman que se trata de dimensiones culturales diferentes. La pauta de transmisión intergeneracional llega incluso a cambiar entre las diversas generaciones políticas: mientras que la legitimidad sigue una pauta de cambio intergeneracional y la desafección otra de continuidad, la insatisfacción se ajusta a la existencia de un efecto período (y carece, por lo tanto, de cualquier efecto cohorte). Desde un punto de vista comparativo, estos resultados sitúan a España en una posición peculiar. Es cierto que, tras la instauración de la democracia, el caso español se enfrenta en la actualidad a retos parecidos a los de otros países europeos occidentales. Pero, aun así, la democracia, española parece caracterizarse por esa inusual combinación de altos grados de legitimidad, que han permanecido estables e inmunes a las fluctuaciones de las percepciones negativas de los ciudadanos sobre el funcionamiento de la democracia, y de niveles igualmente altos de desafección política, también caracterizados por su estabilidad pese a las modificaciones de todo tipo habidas en los entornos políticos, sociales y económicos.

(Traducción de Jesús Cuéllar Menezo.) 


\section{REFERENCIAS BIBLIOGRÁFICAS}

Abramson, Paul R. (1983): Political Attitudes in America. Formation and Change, San Francisco: Freeman and Co.

Almond, Gabriel, y Verba, Sidney (1963): The Civic Culture. Political Attitudes and Democracy in Five Nations, Princeton: Princeton University Press.

Aguilar, Paloma (1995): Memoria y olvido de la guerra civil española, Madrid: Alianza.

Andersson, Christopher J., y Guillory, Christine A. (1997): «Political Institutions and Satisfaction with Democracy: A Cross-National Analysis of Consensus and Majoritarian Systems", American Political Science Review, 91: 66-81.

BALCH, George I. (1974): «Multiple Indicators in Survey Research: The Concept "Sense of Political Efficacy"', Political Methodology, 1: 1-43.

Beetham, David (1991): The Legitimation of Power, Atlantic Highlands, NJ: Humanities Press International.

Bermeo, Nancy (1992): "Democracy and the Lessons of Dictatorship», Comparative Politics, 24: 273-291.

Citrin, Jack (1972): Political Disaffection in America: 1958-1968, tesis doctoral, Universidad de California en Berkeley.

- (1974): «Comment: The Political Relevance of Trust in Goverment», American Political Science Review, 68: 973-988.

Citrin, Jack, y Elters, David J. (1975): Political Disaffection Among British University Students. Concepts, Measurements, and Causes, Berkeley: Institute of International Studies, Universidad de California.

Citrin, Jack; McClosky, Herbert; Shanks, J. Mervill, y Sniderman, Paul M. (1975): «Personal and Political Sources of Political Alienation», British Journal of Political Science, 5: 1-31.

Clarke, Harold D.; DutT, Nitish, y Kornberg, Allan (1993): «The Political Economy of Attitudes toward Polity and Society in Western European Democracies», The Journal of Politics, 55: 998-1021.

Craig, Stephen C.; Niemi, Richard G., y Silver, Glenn E. (1990): «Political Efficacy and Trust: A Report on the NES Pilot Study Items», Political Behavior, 12: 289-314.

Crozier, Michael; Huntington, Samuel P., y Watanuki, Joji (1975): The Crisis of Democracy. Report on the Governability of Democracies to the Trilateral Commission, Nueva York: New York University Press.

DAHL, Robert A. (1971): Polyarchy. Participation and Opposition, New Haven: Yale University Press.

Dalton, Russell J. (1980): «Reassessing Parental Socialization: Indicator Unreliability versus Generational Transfer", American Political Science Review, 74: 421-431.

- (1988): Citizen Politics in Western Democracies. Public Opinion and Political Parties in the United States, Great Britain, West Germany, and France, Chatham, NJ: Chatham House Publishers.

Dalton, Russell J.; Flanagan, Scott C., y Beck, Paul A. (eds.) (1994): Electoral Change in Advanced Democracies: Realignment or Dealignment?, Princeton: Princeton University Press.

Dahrendorf, Ralph (1980): «Effectiveness and Legitimacy: On the "Governability of Democracies"”, The Political Quarterly, 51: 393-410.

Di Palma, Giuseppe (1970): Apathy and Participation. Mass Politics in Western Societies, Nueva York: The Free Press.

- (1990): To Craft Democracies. An Essay on Democratic Transitions, Berkeley: University of California Press.

Diamond, Larry (1998): «Political Culture and Democratic Consolidation», Madrid: Instituto Juan March, Estudio/Working Paper 123.

DiAmOnd, Larry, y LipSeT, Seymour M. (1995): "Legitimacy», en The Encyclopedia of Democracy, ed. Seymour M. Lipset, Londres: Routledge, vol. III.

EAston, David (1965): A Systems Analysis of Political Life, Chicago: The University of Chicago Press. 
EAston, David (1975): «A Re-Assessment of the Concept of Political Support», Journal of Political Science, 5: 435-457.

Farah, Barbara G.; BARnes, Samuel H. y HeunKs, Felix (1979): «Political Dissatisfaction», en Political Action. Mass Participation in Five Western Democracies, Samuel H. Barnes, Max Kaase et al., Beverly Hills: Sage.

Finkel, Steven E.; Muller, Edward N., y Seligson, Michael A. (1989): «Economic Crisis, Incumbent Performance and Regime Support: A Comparison of Longitudinal Data from West Germany and Costa Rica», British Journal of Political Science, 19: 329-351.

FuCHS, Dieter (1992): "Trends of Political Support in the Federal Republic of Germany", en Political Culture in Germany, ed. D. Berg-Scholosser y R. Rytlewski, Nueva York: St. Martin's Press.

FuchS, Dieter, y Klingemann, Hans-Dieter (1995): «Citizens and the State: A Relationship Transformed», en Citizens and the State, ed. Hans-Dieter Klingemann y Dieter Fuchs, Oxford: Oxford University Press.

Fuchs, Dieter; Guidorossi, Giovanna, y SvenSSON, Palle (1995): «Support for the Democratic System", en Citizens and the State, ed. Hans-Dieter Klingemann y Dieter Fuchs, Oxford: Oxford University Press.

García Delgado, José L. (ed.) (1990): Economía española de la transición y la democracia, 19731986, Madrid: Centro de Investigaciones Sociológicas.

Gabriel, Oskar W. (1995): "Political Efficacy and Trust», en The Impact of Values, ed. Jan W. van Deth y Elinor Scarbrough, Oxford: Oxford University Press.

- (1996): "The Confidence Crisis in Germany", Ponencia presentada en la conferencia sobre The Erosion of Confidence in Advanced Democracies, Bruselas, 7-9 noviembre.

Gabriel, Oskar W., y VAN Deth, Jan W. (1995): "Political Interest», en The Impact of Values, ed. Jan W. van Deth y Elinor Scarbrough, Oxford: Oxford University Press.

Gamson, William A. (1968): Power and Discontent, Homewood, Ill.: Dorsey Press.

GunTHER, Richard (1986): "El colapso de UCD", en Crisis y cambio: electores y partidos en la España de los años ochenta, ed. Juan J. Linz y José R. Montero, Madrid: Centro de Estudios Constitucionales.

- (1992): Politica y cultura en España, Madrid: Centro de Estudios Constitucionales.

Gunther, Richard; Diamandouros, P. Nikiforos, y Puhle, Hans-Jürgen (eds.) (1995): The Politics of Democratic Consolidation: Southern Europe in Comparative Perspective, Baltimore: The Johns Hopkins University Press.

Habermas, Jürgen (1975): Legitimation Crisis, Boston: Beacon Press.

Hirschman, Albert O. (1982): Shifting Involvements. Private Interest and Public Action, Princeton: Princeton University Press.

Huntington, Samuel H. (1991): The Third Wave. Democratization in the Late Twentieth Century, Norman: University of Oklahoma Press.

Jennings, Kent M., y Niemi, Richard G. (1981): Generations and Politics: A Panel Study of Young Adults and Their Parents, Princeton: Princeton University Press.

KAASE, Max (1994): «Political Culture and Political Consolidation in Central and Eastern Europe», en Research on Democracy and State, vol. 2, ed. Frederick D. Weil, Greenwich: JAI Press.

KaAse, Max, y Newton, Kent (1995): Beliefs in Government, Oxford: Oxford University Press.

Kinder, Donald R., y Sears, David O. (1985): «Public Opinion and Political Action», en The Handbook of Social Psychology, ed. Gardner Lindzey y Elliot Aronson, Nueva York: Random House, vol. II.

KING, Anthony (1975): “"Overload”: Problems of Governing in the 1970s», Political Studies, 23: 284-296.

Kornberg, Allan, y Clarke, Harold D. (1992): Citizens and Community. Political Support in a Representative Democracy, Cambridge: Cambridge University Press.

Kuechler, Manfred (1991): "The Dynamics of Mass Political Support in Western Europe: Methodological Problems and Preliminary Findings», en Eurobarometer. The Dynamics of European Public Opinion, eds. Karlheinz Reif y Ronald Inglehart, Londres: Macmillan. 
Lagos, Marta (1997): «Latin America’s Smiling Mask», Journal of Democracy, 8: 125-135.

LINZ, Juan J. (1978a): «Crisis, Breakdown and Reequilibration», en The Breakdown of Democratic Regimes, ed. Juan J. Linz y Alfred Stepan, Baltimore: The Johns Hopkins University Press.

- (1978b): «Legitimidad y eficacia en la evolución de los regímenes políticos», en Problemas del subdesarrollo, Granada: Caja General de Ahorros y Monte de Piedad de Granada.

- (1988): «Legitimacy of Democracy and the Socioeconomic System», en Comparing Pluralist Democracies, ed. Mattei Dogan, Boulder: Westview Press.

LinZ, Juan J., y STEPAN, Alfred (1989): «Political Crafting of Democratic Consolidation or Destruction: European and South American Comparisons", en Democracy in the Americas: Stopping the Pendulum, ed. Robert A. Pastor, Nueva York: Holmes and Meier.

- (1996): The Problems of Democratic Transition and Consolidation: Southern Europe, South America, and Post-Communist Europe, Baltimore: The Johns Hopkins University Press.

Lipset, Seymour M. (1981): Political Man: The Social Bases of Politics, edición ampliada, Baltimore: The Johns Hopkins University Press.

Lipset, Seymour M., y SCHNEIDER, William (1983): The Confidence Gap, Nueva York: The Free Press.

LÓpez PINTOR, Rafael (1982): La opinión pública española del franquismo a la democracia, Madrid: Centro de Investigaciones Sociológicas.

- (1987): «El impacto del autoritarismo en la cultura política. La experiencia española en una perspectiva comparada», en Política y sociedad. Homenaje a Francisco Murillo Ferrol, Madrid: Centro de Investigaciones Sociológicas.

- (1997): «Descontento político y tendencias electorales en Europa», en Tendencias de futuro en la sociedad española. Primer foro sobre tendencias sociales, ed. José Félix Tezanos, José Manuel Montero y José Antonio Díaz, Madrid: Sistema.

Mannheim, Karl (1952): Essays on the Sociology of Knowledge, Londres: Routledge and Kegan Paul.

Maravall, José María (1984): La política de la transición, Madrid: Taurus, 2. a ed.

- (1995): Los resultados de la democracia, Madrid: Alianza.

MARAVALL, José María, y SANTAMARía, Julián (1989): «Transición política y consolidación de la democracia española», en La transición democrática española, ed. José F. Tezanos, Ramón Cotarelo y Andrés de Blas, Madrid: Sistema.

McDonough, Peter; Barnes, Samuel H., y López Pina, Antonio (1986): «The Growth of Democratic Legitimacy", American Political Science Review, 80: 735-760.

- (1992): "The Nature of Political Support and Legitimacy in Spain», Comparative Political Studies, 27: 349-380.

Miller, Arthur H. (1974a): «Political Issues and Trust in Government: 1964-1970», American Political Science Review, 68: 951-972.

- (1974b): «Rejoinder to "Comment" by Jack Citrin: Political Discontent or Ritualism», American Political Science Review, 68: 989-1001.

Mishler, William, y Rose, Richard (1996): «Trajectories of Fear and Hope. Support for Democracy in Post-Communist Europe», Comparative Political Studies, 28: 553-581.

MoIsÉs, José Álvaro (1995): Os brasileiros e a democracia. Bases sócio-políticas de legitimidade democrática, San Pablo: Ática.

Montero, José Ramón (1993a): «Revisiting Democratic Success: Legitimacy and the Meanings of Democracy in Spain", en Politics, Society, and Democracy. The Case of Spain, ed. Richard Gunther, Boulder: Westview.

- (1993b): «Las dimensiones de la secularización: religiosidad y preferencias políticas en España", en Religión y sociedad en España, ed. Rafael Díaz-Salazar y Salvador Giner, Madrid: Centro de Investigaciones Sociológicas.

Montero, José Ramón, y TorCaL, Mariano (1990): «Voters and Citizens in a New Democracy: Some Trend Data on Political Attitudes in Spain», International Journal of Public Opinion Research, 2: 116-140. 
MONTERO, José Ramón, y MorLINO, Leonardo (1993): «Legitimidad y democracia en el Sur de Europa», REIS, 64: 7-40.

Montero, José Ramón, y Gunther, Richard (1994): «Democratic Legitimacy in Spain», Ponencia presentada en el Congreso Mundial de la Asociación Internacional de Ciencia Política, Berlín.

MonZÓN, Cándido (1988): «La transformación de la cultura política de los españoles», Documentación Social, 73: 103-122.

Morlino, Leonardo (1985): Cómo cambian los regímenes políticos. Instrumentos de análisis, Madrid: Centro de Estudios Constitucionales.

Morlino, Leonardo, y Montero, José Ramón (1995): «Legitimacy and Democracy in Southern Europe», en The Politics of Democratic Consolidation. Southern Europe in Comparative Perspective, ed. Richard Gunther, P. Nikiforos Diamandouros y Hans-Jürgen Puhle, Baltimore: The Johns Hopkins University Press.

Morlino, Leonardo, y TARCHI, Marco (1996): «The Dissatisfied Society: The Roots of Political Change in Italy», European Journal of Political Research, 30: 41-63.

Muller, Edward. W., y Jukam, Thomas O. (1977): «On the Meaning of Political Support», American Political Science Review, 71: 1561-1595.

Muller, Edward N.; Jukam, Thomas O., y Seligson, Martin A. (1982): «Diffuse Support and Anti-system Political Behaviour: A Comparative Analysis», American Journal of Political Science, 26: 240-263.

O'Connor, James (1973): The Fiscal Crisis of the State, Nueva York: St. Martin's Press.

O’Donnell, Guillermo, y SCHMitTer, Philippe C. (1986): Transitions from Authoritarian Rule: Tentative Conclusions About Uncertain Democracies, Baltimore: The Johns Hopkins University Press.

OfFe, Claus (1984): «Legitimacy versus Efficiency», en Contradictions of the Welfare State, Cambridge: Massachusetts Institute of Technology Press.

Pridham, Geoffrey (1995): «The International Context of Democratic Consolidation: Southern Europe in Comparative Perspective», en The Politics of Democratic Consolidation Southern Europe in Comparative Perspective, ed. Richard Gunther, P. Nikiforos Diamandorous y Hans-Jürgen Puhle, Baltimore: The Johns Hopkins University Press.

Putnam, Robert (1993): Making Democracy Work. Civic Traditions in Modern Italy, Princeton: Princeton University Press.

Pye, Lucian W. (1971): "The Legitimacy Crisis», en Crisis and Sequences in Political Development, ed. Leonard Binder et al., Princeton: Princeton University Press.

RAWLS, John (1993): Political Liberalism, Nueva York: Columbia University Press.

Rose, Richard (ed.) (1980): Challenge to Governance, Beverly Hills: Sage.

Rose, Richard, y Haerpfer, Christian (1992): «New Democracies Between State and Market. A Baseline Report of Public Opinion", en Studies in Public Policy, Glasgow: Centre for the Study of Public Policy, University of Strathclyde.

Rose, Richard, y Mishler, William (1996): "Testing the Churchill Hypothesis: Popular Support for Democracy and its Alternatives», Journal of Public Policy, 16: 29-58.

- (1997): «Political Patience in Regime Transformation: A Comparative Analysis of PostCommunist Citizens», en Studies in Public Policy, Glasgow: Centre for the Study of Public Policy, University of Strathclyde.

Rose, Richard, y SHIN, Doh C. (1997): «Discerning Qualities of Democracy in Korea and Postcommunist Countries». Manuscrito.

SANI, Giacomo (1992): «Comportamientos de masas y modelos de ciudadanos», Revista del Centro de Estudios Constitucionales, 13: 127-150.

SchmitT, Hermann (1983): «Party Government in Public Opinion: A European Cross-National Comparison", European Journal of Political Research, 11: 353-375

SHIN, Doh C., y SHYU, Huoyan (1997): «Political Ambivalence in South Korea and Taiwan», Journal of Democracy, 8:110-124. 
SHIN, Doh C., y Rose, Richard (1997): «Koreans Evaluate Democracy: A New Korea Barometer Survey», en Studies in Public Policy, Glasgow: Centre for the Study of Public Policy, University of Strathclyde.

Schuman, Howard, y ScOTT, Jacqueline (1989): «Generations and Collective Memories», American Sociological Review, 54: 359-381.

TOKA, Gábor (1995): «Political Support in East-Central Europe», en Citizens and the State, ed. Hans-Dieter Klingemann y Dieter Fuchs, Oxford: Oxford University Press.

TOPF, Richard (1995): «Beyond Electoral Participation», en Citizens and State, ed. Hans-Dieter Klingemann y Dieter Fuchs, Oxford: Oxford University Press.

TORCAL, Mariano (1995): Actitudes politicas y participación en España. Pautas de cambio y continuidad, tesis doctoral, Madrid: Departamento de Ciencia Política, Universidad Autónoma de Madrid.

Van Deth, Jan W. (1990): "Interest in Politics», en Continuities in Political Action, ed. M. Kent Jennings, Jan W. van Deth et al., Berlín: Walter de Gruyter.

- (1991): «Politization and Political Interest», en Eurobarometer: The Dynamics of European Public Opinion, ed. Karlheinz Reif y Ronald Inglehart, Londres: Macmillan.

WeIL, Frederick D. (1987): "Cohorts, Regimes, and the Legitimation of Democracy: West Germany since 1945", American Sociological Review, 52: 308-324.

- (1989): «The Sources and Structure of Legitimation in Western Democracies: A Consolidated Model Tested with Time-Series Data in Six Countries Since World War II", American Sociological Review, 54: 684-706.

- (1993): «The Development of Democratic Attitudes in Eastern and Western Germany in a Comparative Perspective», Research on Democracy and Society, vol. 1, ed. Frederick D. Weil, Greenwich: JAI Press.

WERT, José Ignacio (1996): «Sobre cultura política: legitimidad, desafección y malestar», en Entre dos siglos. Reflexiones sobre la democracia española, ed. Javier Tusell, Emilio Lamo de Espinosa y Rafael Pardo, Madrid: Alianza.

Wiarda, Howard J. (1989): The Transition to Democracy in Spain and Portugal, Washington, DC: The American Enterprise Institute.

\section{ABSTRACT}

This paper examines the main attitudes towards democracy in Spain in the last two decades. Numerous empirical indicators have been selected to differentiate among three facets which tend to be considered indiscriminately and therefore systematically confused: democratic legitimacy, political discontent and political disaffection. This paper analyses their respective evolution since the transition to democracy and contends that they belong to different conceptual and empirical dimensions. To this end, the authors include the results of two types of analysis: a factor analysis which confirms the different grouping of the indicators at individual level, and a cohort analysis which identifies the differential patterns of continuity and change in six generations spaniards. 